ISSN: 0514-7336

DOI: http://dx.doi.org/10.14201/zephyrus201474203223

\title{
EN TORNO A LA RED ROMANA DE ABASTECIMIENTO DE AGUA A TOLEDO: EXCAVACIONES EN LOS TERRENOS DE LA ACADEMIA MILITAR DE INFANTERÍA
}

\section{Roman water supply to Toledo (Spain): excavations in the fields of the Military Academy of Infantry}

\author{
Marisa Barahona Oviedo*, Miguel Arenillas Parra** y Juan Manuel Rojas Rodríguez-Malo*** \\ * Dpto. de Prehistoria y Arqueología. Facultad de Geografía e Historia, UNED. C/ Senda del Rey, 7. 28040 Madrid. \\ Correo-e: barahonamarisa@gmail.com \\ ** ETS de Ingenieros de Caminos, Canales y Puertos-UPM. C/ Profesor Aranguren, 3. 28040 Madrid. Correo-e: \\ map@ing75.com. \\ *** Juan Manuel Rojas-Arqueología S.L. Cl Taller del Moro, 7, esc. 3, bajo dcha. 45002 Toledo. Correo-e: : jmro- \\ jasrodriguez@yahoo.es
}

Recepción: 27/06/2014; Revisión: 26/07/2014; Aceptación: 15/09/2014

BIBLID [0514-7336 (2014) LXXIV, julio-diciembre; 203-223]

\begin{abstract}
Resumen: Las excavaciones arqueológicas llevadas a cabo en torno a los restos del acueducto que atraviesa los terrenos del campo de maniobras de la Academia Militar de Infantería de Toledo han permitido conocer con detalle la traza de uno de los sistemas hidráulicos que abastecieron a la ciudad romana. El estudio de esta obra se centró además en definir pormenorizadamente su forma constructiva y características técnicas -pendiente y volúmenes portables-, lo que permite diferenciar netamente a este acueducto del procedente de la presa de la Alcantarilla, también vinculado con el sistema de abastecimiento a la antigua Toletum. Queda así patente que nos hallamos ante dos conducciones diferentes, que deben unirse, no obstante, en las cercanías de la ciudad. Dentro del recorrido de la conducción excavada se construyó la torre hoy conocida como el Horno de Vidrio, un elemento singular por sus características dentro de la ingeniería hidráulica romana. La situación de esta estructura facilitaba al acueducto un trazado menos abrupto hacia Toledo, a la vez que permitía la pérdida de carga en un canal de pendiente excesiva.

Palabras clave: Toletum; Acueductos; Ingeniería hidráulica romana; Sistemas de pérdida de carga.

ABSTRACT: Archaeological works carried out in connection with the aqueduct located in the fields of the Military Academy of Infantry of Toledo have allowed us to know new and interesting data about the Roman water supply to the ancient city of Toletum. These excavations were focused on reporting the route, design and technical characteristics -gradients and capacity-. Every data obtained show that the channel through the lands of the Academy is distinctly different of the Roman aqueduct from the Alcantarilla dam, although we supposed that both of them should join in some point near the city.

The pressure drop tower popularly known as the Horno de Vidrio is located in the final section of the aqueduct that has been studied. It is a very singular structure with double aim: first, to facilitate the aqueduct a less steep path towards Toledo. Second, to lose height in an aqueduct with excessive slope.

Key words: Toletum; Aqueducts; Roman hydraulic engineering; Pressure drops devices.
\end{abstract}




\section{Introducción}

Durante los meses de verano del año 2010 se llevaron a cabo intervenciones arqueológicas en el tramo final de uno de los acueductos que abastecieron de agua a la ciudad de Toledo en época romana, cuya traza discurre a través de los terrenos del campo de maniobras Los Alijares de la Academia Militar de Infantería de Toledo ${ }^{1}$.

La realización de estos trabajos permitió la definición de la obra en un recorrido de más de 2,5 $\mathrm{km}$, pudiendo documentarse su forma constructiva y diversas reparaciones llevadas a cabo durante su periodo de funcionamiento. Dentro del tramo intervenido se localiza la torre de pérdida de carga conocida desde antiguo como el Horno de Vidrio, cuyo análisis ha aportado asimismo datos novedosos, en especial en lo que concierne a los criterios técnicos que guiaron la construcción del acueducto en el tramo estudiado. Por último, las conclusiones alcanzadas ratifican la existencia de una red de abastecimiento de agua a la antigua Toletum más compleja que la que se admitía tradicionalmente, según ya se expuso en un trabajo anterior (Arenillas et al., 2009a). Al mismo tiempo, los resultados obtenidos corrigen algunas de las propuestas entonces defendidas, en concreto las relativas al trazado del tramo final de dos de los tres acueductos recientemente identificados para el abastecimiento a la ciudad: el de la Rosa y el de la Alcantarilla.

La intervención realizada combinó la excavación arqueológica en numerosos puntos de la conducción con la prospección intensiva del terreno. La localización exacta, dimensiones y el número final de sondeos realizados -más de 50- hubo de establecerse en numerosas ocasiones sobre el terreno, en función de la ubicación de los restos del canal previamente conocidos y a partir de los resultados

${ }^{1}$ La intervención fue financiada por la Confederación Hidrográfica del Tajo (Ministerio de Agricultura, Alimentación y Medio Ambiente) como continuación de los estudios que sobre el abastecimiento de agua a Toledo en época romana ha abordado en los últimos años dicho organismo (Arenillas et al., 2009a). Los trabajos fueron coordinados por M. Arenillas Parra. La dirección arqueológica corrió a cargo de $\mathrm{M}$. L. Barahona y J. M. Rojas, con la colaboración del también arqueólogo A. Vicente. Actualmente M. L. Barahona disfruta de una beca predoctoral de la Fundación Juanelo Turriano. Agradecemos a la Academia Militar de Infantería de Toledo las facilidades brindadas para llevar a cabo este estudio.

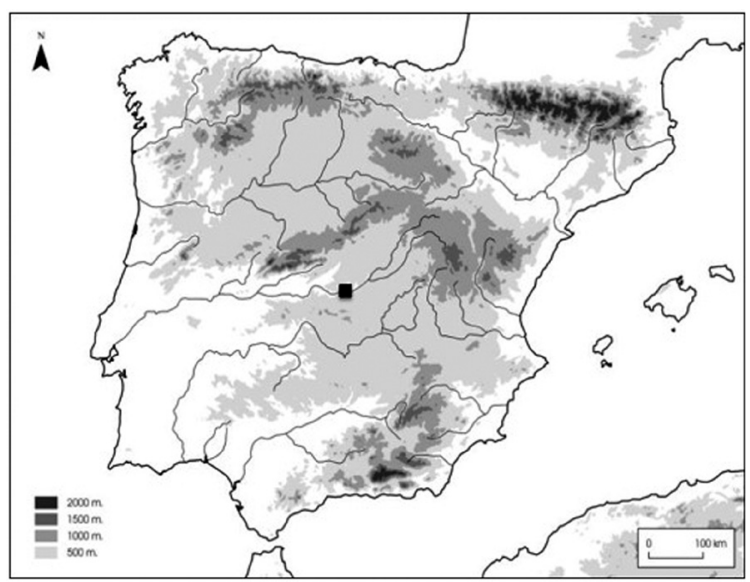

Fig. 1. Localización de Toletum en la Península Ibérica.

de las propias excavaciones que se iban efectuando. Esta estrategia de trabajo estuvo condicionada por la dificultad para definir en la actualidad el trazado concreto del acueducto, enterrado en la mayor parte de su recorrido. Además de ello, algunas de las variables topográficas a las que tuvo que adaptarse la obra en el momento de su construcción han sufrido notables transformaciones hasta configurarse la morfología actual del terreno, lo que ha dificultado aún más su seguimiento. En todos los casos el registro de datos se llevó a cabo por niveles de estratigrafía arqueológica, según el método Harris. La misma metodología se empleó en la excavación del Horno de Vidrio, aunque no llegaron a alcanzarse los niveles naturales en el sector o del interior de la torre para evitar posibles problemas de estabilidad de la estructura.

La sistematización por sondeos de la intervención realizada permitió la identificación y documentación del recorrido de la conducción romana en una importante longitud -más de $2,5 \mathrm{~km}$, según se ha dicho-, obteniéndose además datos suficientes para determinar algunas características técnicas como su pendiente y capacidad. En contrapartida, la ausencia de relaciones estratigráficas directas entre los diferentes sondeos merma las posibilidades de secuenciar las reparaciones identificadas en la conducción, así como su relación con la torre acuaria del Horno de Vidrio. La tipología se revela en este caso como la herramienta principal para elaborar la secuencia constructiva del acueducto. 


\section{De la teoría del acueducto único a la propuesta de una red compleja de abastecimiento de agua a Toletum: historiografía}

Los estudios realizados hasta hace una década sobre el suministro de agua a Toledo en época romana contemplaban la existencia de un único aunque importante sistema para proveer a la ciudad, cuyo trazado era, en gran parte, teórico ${ }^{2}$.

El origen de esta hipótesis se remonta a mediados del s. XviII y debe enmarcarse dentro de los trabajos de catalogación de las antigüedades nacionales que impulsó la Corona española bajo Fernando vi. Los encargados de coordinar tan magna tarea fueron los jesuitas A. Marcos Burriel y F. Pérez Bayer, quienes centralizaron parte de su trabajo en Toledo. Entre los ańos 1752 y 1753 , y dentro del marco descrito, estos religiosos documentaron, junto con el académico Palomares, diversos restos de construcciones hidráulicas distribuidos a lo largo de dos extensas áreas entre los Montes de Toledo y la ciudad, que identificaron como pertenecientes una misma obra romana $^{3}$ (Ponz, [1787] 1972, I: 209-211; Porres, 1984). En su primer tramo, esta supuesta conducción tomaría sus aguas de los arroyos de la sierra del Castañar, en los Montes de Toledo, conociéndose diversos restos de su fábrica hasta la población de Layos. Dentro de este recorrido se integraban las ruinas de la propia presa de la Alcantarilla, considerada entonces como una gran obra de paso arruinada sobre el arroyo Guajaraz ${ }^{4}$. Tras un gran vacío de casi nueve kilómetros en línea recta, un segundo conjunto de restos se agrupaba en la dehesa de la Sisla, al se de la ciudad -actualmente parte de los terrenos de la Academia de Infantería de Toledo-. Además de varios tramos de canal y el puente-acueducto sobre el Tajo, Pérez Bayer localizaba en este espacio dos torres acuarias -castellum-, siendo la más cercana a la ciudad el llamado Horno de Vidrio, y desconociéndose en la actualidad la localización de la segunda. Señala también el jesuita un nuevo punto de captación, además del ya indicado en los Montes de Toledo, al s del monasterio de la

\footnotetext{
2 Una revisión historiográfica más completa puede encontrarse en Barahona, 2009: 61-94.

3 Pérez Bayer, F.: De Toletano Hebraeorum Templo, 1752. Biblioteca Pública de Toledo, Toledo (BPT), manuscrito 128.

${ }^{4}$ La presa no fue interpretada como tal hasta principios del s. xx (López de Ayala, [1905] 1959: 174).
}

Sisla, junto al arroyo de la Degollada y al camino de Cobisa, en una fuente "que nace a borbollones en bastante copia".

Casi dos siglos después, en 1948, el ingeniero Ángel Ortiz Dou realizó una propuesta teórica de trazado para el acueducto que integraba las distintas estructuras documentadas en el s. XVIII ${ }^{5}$. En esta nueva interpretación la cabecera del sistema no se situaba en las fuentes de la sierra del Castañar, sino en la ya identificada presa de la Alcantarilla. El canal que parte de la misma era unido de forma teórica con los restos de construcciones hidráulicas de la dehesa de la Sisla y el paso sobre el Tajo. Finalmente, los restos de conducción romana conocidos en la sierra del Castañar con origen en el arroyo de San Martín de la Montińa fueron explicados por Ortiz Dou como un trasvase de cuencas para incrementar los volúmenes de agua que se recogerían en el embalse de la Alcantarilla. La propuesta de este autor, nunca contrastada, fue aceptada con generalidad por todos los estudiosos del tema hasta casi la actualidad (Porres, 1970; Fernández Casado, 1972 y 1983: 127-135, 255, 515 y 535; Celestino, 1976; Aranda, Carrobles e Isabel, 1997; Arenillas et al., 1999), pese a las dificultades técnicas que encierra (Arenillas et al., 2009b: 143-146; Arenillas, 2009: 193-194). El segundo punto de captación citado por Pérez Bayer en la Sisla, al que Ortiz Dou hace referencia de soslayo, quedó relegado al olvido.

"Resuelto" el problema del trazado del acueducto, y junto con los estudios que se llevan a cabo desde principios de siglo, fundamentalmente monográficos sobre la presa o el paso de la conducción sobre el Tajo (Amador de los Ríos, 1905: 21-24; Rey Pastor, 1928: 168-170; Porres, 1970; Celestino, 1976; Sánchez Abal, 1977 o Fernández Casado, 1961, 1972 y 1983: $127-135$ y 515), surgirá el interés por el estudio de la red de distribución del agua en el interior de la ciudad y la localización del depósito terminal de la conducción (Fernández Casado, 1961: 357, 1972, 1977a y 1977b; García-Diego, 1974 y 1975; Porres, 1975; Sáenz, 1977; García-Diego y Porres, 1977; Aranda, Carrobles e Isabel, 1997: 165-270 y 330-334). Estos últimos estudios serán completados con posterioridad, al compás de la arqueología urbana toledana, que ha permitido la excavación de diferentes estructuras

5 Aunque este trabajo fue editado por el Ministerio de Obras Públicas sin autor explícito, el propio Ortiz Dou indicaba su autoría en una publicación anterior (1947: 285). 


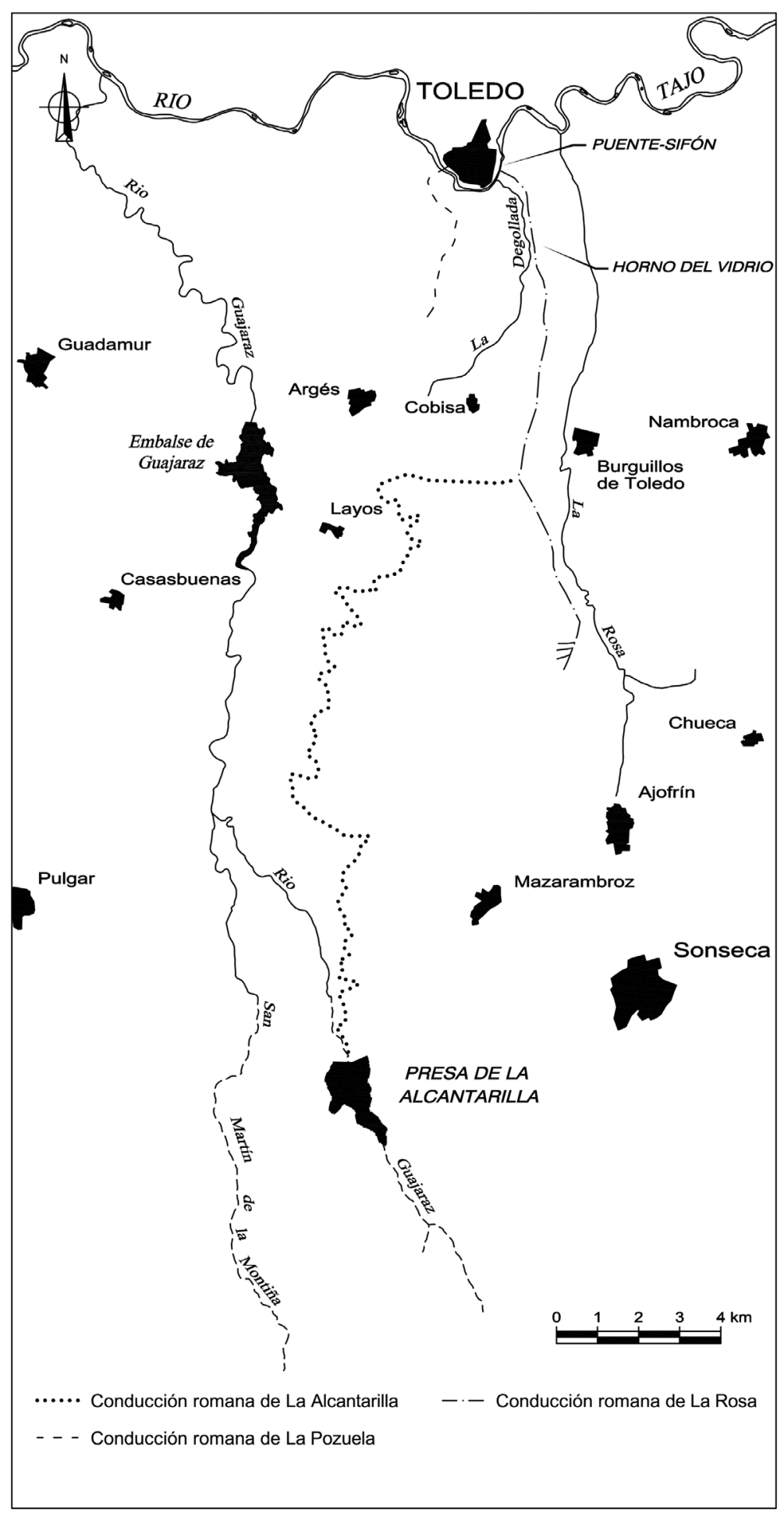

FIG. 2. Propuesta de trazado de la red romana de abastecimiento de agua a Toletum (Arenillas et al., 2009a: 123). hidráulicas relacionadas con el almacenamiento, distribución y evacuación de agua en la antigua urbe (fundamentalmente, Porres, 1983; Lavado y Martín, 1986; Lavado, 1988; Prieto, 1990; Sánchez-Palencia, 1996; Sánchez-Chiquito, 2005; Rojas et al., 2007; Ruiz Taboada y Arribas, 2007; Arribas, 2008), aunque apenas existen estudios actualizados de conjunto (Cauce, 2009: 32-34). En cuanto a la cronología de esta amplia infraestructura, se acepta con generalidad su funcionamiento entre los ss. I y IV d. C., fechas coherentes con la obtención del rango de municipalidad de Toletum y con la amortización de los elementos excavados en el interior de la ciudad (Aranda, Carrobles e Isabel, 1997: 334336; Rubio, 2005).

Entre los años 2005 y 2009, la Confederación Hidrográfica del Tajo llevó a cabo una serie de estudios sobre el abastecimiento romano de aguas a Toledo (Arenillas et al., 2009a). La realización de estos trabajos, que combinaron la prospección en superficie, técnicas geofísicas - georradar- y sondeos arqueológicos, proporcionó nuevos y numerosos datos sobre la red de abastecimiento de agua a la ciudad romana, que suponían un cambio radical en la tradicional concepción del sistema de suministro a la antigua Toletum. Así, frente a la histórica asignación de un único acueducto a la urbe desde la presa de la Alcantarilla, se propuso la existencia de una red mucho más compleja, compuesta al menos por tres sistemas de captación de agua: La Rosa, La Alcantarilla y La Pozuela; acorde, por otra parte, con la realidad conocida en otras grandes ciudades del Imperio en Hispania. Además de ello, se demostró la imposibilidad de conexión entre los restos del supuesto trasvase de San Martín de la Montiña y el embalse de la Alcantarilla y, por tanto, la desvinculación del primero con el abastecimiento de agua al Toledo romano. 
Según la propuesta entonces defendida (Arenillas et al., 2009b: 149-172; Arenillas, 2009: 205207), el primero de estos sistemas en el tiempo habría sido el procedente del arroyo de la Rosa. Su traza pudo verificarse con dirección s-N en paralelo a este curso y casi desde su origen ${ }^{6}$ hasta el área conocida como Los Torrejones-Olivar de Burguillos, ya en el interior del campo de maniobras militar. Siguiendo dicha interpretación, las aguas de este acueducto discurrirían en galería hasta la ciudad y formarían también parte de este sistema tanto los restos de la torre del Horno de Vidrio en la dehesa de la Sisla, como el puente-acueducto sifón sobre el río Tajo. En un segundo momento, en torno a la segunda mitad del s. I d. C., se habrían construido la presa y la conducción de la Alcantarilla. El trazado de esta última puede seguirse en superficie hasta la población de Layos donde, tras un túnel de aproximadamente $3 \mathrm{~km}$ de longitud identificado con técnicas geofísicas -georradar-, que discurre de E a O bajo las estribaciones septentrionales de la Sierra de Layos, se uniría con la galería de la Rosa.

La tipología en túnel que presentaba presumiblemente el acueducto principal, fruto de la unión de los sistemas de la Rosa y la Alcantarilla, nos llevó a desestimar como parte de esta obra dos tramos de conducción en superficie conocidos desde antiguo en la dehesa de la Sisla, en las laderas occidentales del Cerro de los Alcaravanes y el Cerro Cortado ${ }^{7}$ (Fernández Miranda, Mangas y Plácido, 1990: 33; Aranda, Carrobles e Isabel, 1997: 293-294). Se planteó así la posibilidad de que estos restos perteneciesen a una conducción secundaria, procedente del Arroyo de la Degollada, que aportaría caudales adicionales al acueducto principal y habría podido construirse tras la pronta rotura de la presa de la $\mathrm{Al}-$ cantarilla (Arenillas et al., 2009b: 166-167 y 170; Arenillas 2009: 205-207). Causas similares podrían haber dado lugar a la construcción de la galería de captación-conducción en la dehesa de la Pozuela, en

${ }^{6}$ Se apuntó entonces como cabecera de este abastecimiento el límite entre los términos de Burguillos y Ajofrín, donde existe una zona geológicamente apta para la surgencia de un manantial. Recientemente hemos tenido noticia de una serie de galerías subterráneas en el término de Ajofrín, a unos $3 \mathrm{~km}$ al s de la zona de captación por nosotros propuesta, que bien podrían estar también vinculadas con el caput aquae de esta conducción.

7 вРт, 1752, ms. 128. uso hasta mediados del siglo pasado. La constatación documental de la existencia de este sistema con anterioridad al s. XVI y las características tipológicas de algunas de sus fases constructivas nos llevaron a proponer igualmente su vinculación con el abastecimiento de agua a la ciudad de Toledo en época romana, y su construcción posterior al colapso de la presa de la Alcantarilla (Barahona et al., 2007; Arenillas et al., 2009b: 172-185; Arenillas, 2009: 205-207).

La multiplicación de datos relativos a la antigua red de suministro hídrico a la urbe hacían necesario matizar y profundizar algunas de las cuestiones planteadas con nuevos trabajos arqueológicos que permitiesen confirmar algunas de las hipótesis expuestas que contaban con menor respaldo de restos materiales. Se dispuso de este modo la realización de las intervenciones recogidas en estas páginas, cuyos objetivos principales se enfocaron en los restos localizados en los terrenos del campo de maniobras de la Academia de Infantería de Toledo, con tres vertientes de investigación: a) confirmación del trazado del tramo de conducción conjunta de la Rosa-la Alcantarilla a través de los terrenos de la Academia militar y hasta su paso sobre el Tajo; b) documentación del trazado del canal en superficie considerado un ramal secundario y cuyos restos se localizaban tanto en el Cerro de los Alcaravanes como en el Cerro Cortado; c) estudio de la torre del Horno de Vidrio, cuyo primer análisis parecía determinar al menos dos fases de uso en la estructura (Arenillas et al., 2009: 168-169).

\section{La conducción romana del campo de maniobras Los Alijares de Toledo}

Las excavaciones y prospecciones arqueológicas llevadas a cabo en el campo de maniobras de la Academia Militar de Infantería de Toledo han permitido documentar parte del trazado de uno de los acueductos que abastecieron a la ciudad en época romana. Las características constructivas de los diferentes tramos de conducción en los que se ha intervenido permiten afirmar que nos hallamos ante una misma obra, de no poca entidad, de la que también formaron parte la llamada torre del Horno de Vidrio, los restos ya conocidos de canal en el Cerro Cortado y el Cerro de los Alcaravanes, y el puente-sifón sobre el Tajo, contrariamente a la hipótesis defendida por algunos de nosotros en 


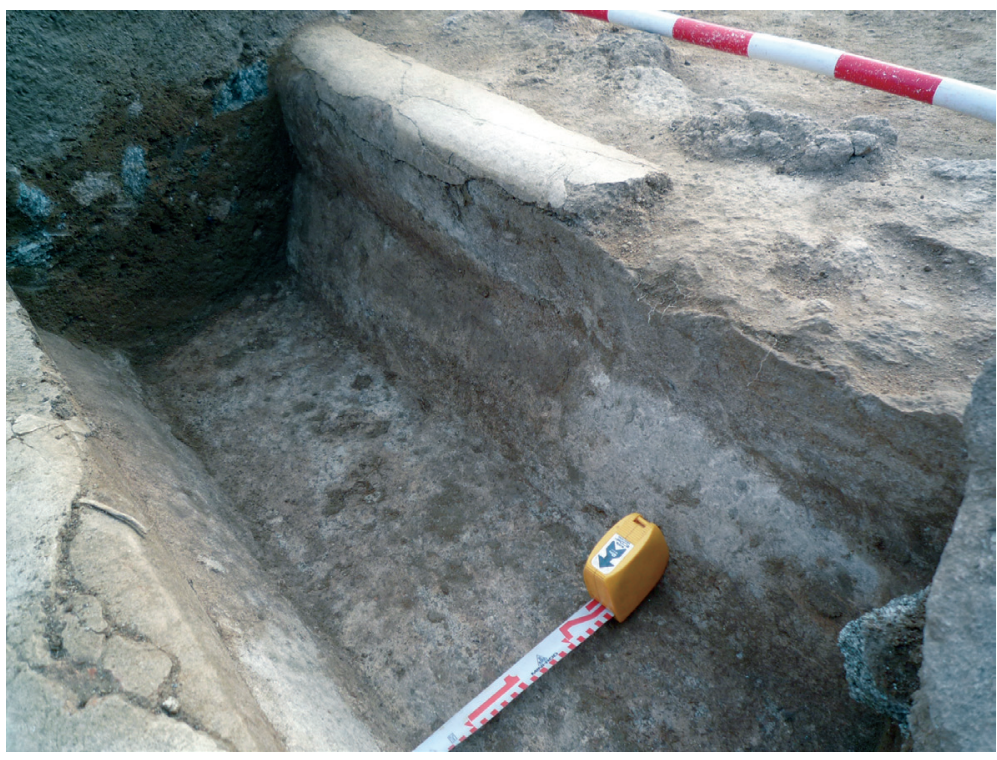

Fig. 3. Excavación de un tramo del acueducto en el Cerro de los Alcaravanes.

\subsection{Caracterización constructiva del canal excavado}

El acueducto discurre en superficie, adaptándose de forma sistemática a la morfología del terreno, salvando sus irregularidades con pequeños cortes para acomodar su estructura o, incluso, excavando la roca hasta $1,5 \mathrm{~m}$ de profundidad cuando es necesario para mantener la cota y pendientes de la conducción. En ocasiones también debió elevarse sobre un muro corrido (substructio) para mantener el nivel, como el que ha podido documentarse en la vaguada de la ladera $\mathrm{E}$ de los Cerros del Horno de Vidrio (sondeo 9.1), aunque la morfología del terreno permite intuir su necesidad en otros puntos.

El canal está compuesto por dos muros cajeros de opus caementicium -en los que se ha documentado el uso

anteriores trabajos (Arenillas et al., 2009b: 158172; Arenillas, 2009).

El acueducto discurre de $\mathrm{s}$ a $\mathrm{N}$ hacia Toledo por la margen derecha del cauce del Arroyo de la Degollada, del que se separa conforme éste se encajona y desciende hacia el Tajo, para situarse en la vertiente o del Cerro de los Alcaravanes, próximo a la divisoria de aguas entre los valles de la Degollada y la Rosa. Desde allí avanza hasta la torre de pérdida de carga del Horno de Vidrio, donde se produce un cambio de cuenca hacia La Rosa, y sigue su recorrido por la falda oriental de los cerros del mismo nombre, discurriendo al o de la antigua Venta de Santa Ana. Por último, la conducción bordea la vertiente occidental del Cerro Cortado hasta situarse en su ladera $\mathrm{N}$, frente a Toledo, donde en esta intervención se han localizado nuevos restos relacionados con el acueducto. Hacia el E, la existencia de un pinar de repoblación plantado sobre antiguas estructuras de época reciente dificulta notablemente la localización de posibles vestigios. Frente al cerro, y a unos $350 \mathrm{~m}$ al $\mathrm{N}$, restos de pilares de opus caementicium conocidos desde antiguo ${ }^{8}$ y, al no de los mismos, las ruinas del venter para el paso en sifón sobre el Tajo completan el trazado conocido del acueducto.

8 BPT, 1752, ms. 128. de fragmentos de cerámica como caementa- que se apoyan sobre una base más ancha también de la misma fábrica. A menudo se observan en sus caras exteriores las líneas de las tablas de encofrado empleadas para su construcción. El espesor de estos cajeros es de $27-30 \mathrm{~cm}$ de media y su altura 40-42 $\mathrm{cm}$. Entre ellos queda libre un espacio variable, de unos $40 \mathrm{~cm}$ como norma, que en ocasiones supera los $50 \mathrm{~cm}^{9}$, lo que supone un ancho medio total de 1-1,10 m. En los restos que bordean la falda occidental de Cerro Cortado -sondeos 12.1 y 12.3- se han comprobado, no obstante, diferencias de hasta casi $10 \mathrm{~cm}$ de espesor entre los cajeros. Esta disparidad podría estar relacionada con el trazado de la obra a lo largo de una ladera muy pendiente, donde el muro interno del canal -el derecho ${ }^{10}$ en este casofunciona realmente como estructura de contención

9 Esta variación puede relacionarse con las zonas de menor pendiente del canal, según se explicará más adelante (cf. apartado 5).

${ }_{10}$ Para la descripción del acueducto, y dado que este presenta diferentes orientaciones al adaptarse al terreno por el que discurre, adoptamos aquí la nomenclatura clásica empleada en hidrología. Así, en este caso, al hablar del lado derecho del canal, nos referimos al que queda a nuestra derecha si miramos a este según la dirección de la corriente, es decir, si miramos hacia aguas abajo. 
de tierras, lo que exige su mayor entidad. En el mismo sentido creemos que debe interpretarse la diferencia de altura de varios centímetros, de nuevo a favor del cajero interior de la obra, documentada en varios de los sondeos efectuados tanto en el Cerro de los Alcaravanes como en el Cerro Cortado -sondeos 6.17, 6.19, 6.21 y 12.1-.

En el espacio entre cajeros, la base del canal está formada por un primer nivel muy compacto, de $4-5 \mathrm{~cm}$ de espesor, realizado con cantos pequeńos y abundante mortero de cal. Sobre el mismo se dispone una segunda capa, compuesta por fragmentos cerámicos de tamaño centimétrico $-0,5-3 \mathrm{~cm}-$ ligados con abundante mortero, que se

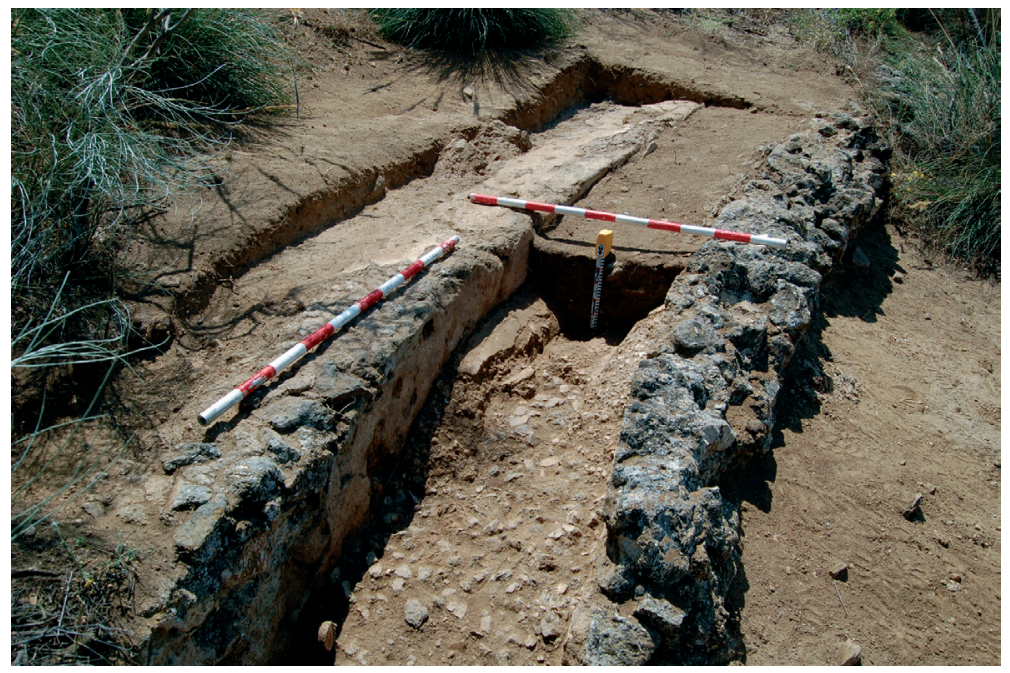

FIG. 4. Excavación de un tramo del acueducto en el Cerro Cortado. eleva además lateralmente por la parte inferior de los muros cajeros, estando engrosada la unión entre los laterales y la base del canal. No se ha podido determinar el espesor exacto de este revestimiento, aunque de algunos datos puntuales se puede deducir que sería, mínimo, de 4 $\mathrm{cm}$. Por último, un acabado muy pulido de mortero, de unos $2 \mathrm{~cm}$ de grosor y realizado con elementos cerámicos machacados de tamaño milimétrico y abundante cal, cubre tanto la base como los lados interiores de la caja del canal, estando sus ángulos inferiores rematados en escocia ${ }^{11}$. Alcanza este revestimiento la parte superior de los cajeros, donde termina en una media caña ${ }^{12}$. Con estas características, la sección interior del canal presenta forma de 'u' abierta, con $32 \mathrm{~cm}$ de altura, $25 \mathrm{~cm}$ de ancho medio en la base y $40 \mathrm{~cm}$ en la parte superior.

11 Algunos autores han señalado, en relación con los restos de canal conocidos en el Cerro de los Alcaravanes, que estas escocias - a las que denominan 'cuñas'- fueron "añadidas posteriormente a la sección de mampostería hormigonada” (Aranda, Carrobles e Isabel, 1997: 294), lo que no ha sido documentado en ninguno de los tramos de la conducción excavada, tratándose en todos los casos de un mismo revestimiento continuo.

12 Las características de los dos últimos revestimientos descritos se corresponden con las del material de construcción romano conocido como opus signinum. Para una mayor agilidad en el texto, y siguiendo la denominación empleada ya por algunos autores (Silva, 2002: 294), pasaremos a denominar opus signinum 'fino' al revoco final pulido y opus signinum 'grosero' al que se sitúa bajo el anterior.
La cubierta del acueducto es, sin duda, uno de los elementos más singulares de la obra. Se trata de grandes piezas de barro cocido, hechas con molde, cuya forma se corresponde en sección con la de una bóveda de medio punto, rematada lateralmente en dos pestañas horizontales para asegurar su apoyo sobre la coronación de los muros de la conducción. Ha podido documentarse en posición primaria en tres de los sondeos realizados -sondeos 6.4, 6.21 y 6.23-, encontrándose numerosos fragmentos de la misma en otros muchos. Mide $75 \mathrm{~cm}$ de anchura -de los cuales $42 \mathrm{~cm}$ corresponden al diámetro de la bóveda-, $30 \mathrm{~cm}$ de longitud y unos $4 \mathrm{~cm}$ de espesor. Las piezas documentadas presentan dobles digitaciones cruzadas en ambas caras y existen diferencias en sus pastas, donde hemos distinguido tres tipologías: pastas oxidantes de color anaranjado oscuro con desgrasantes gruesos; pastas oxidantes de color anaranjado claro con desgrasantes finos; y pastas de cocción mixta con desgrasantes gruesos. Este elemento de cobertura se coloca directamente sobre la coronación de los muros, trabándose las piezas entre sí y con los cajeros con mortero de cal, que también se emplea para tapar grietas o fisuras que por rotura se han formado en algunas de ellas. Se observa asimismo el empleo de ripios - piedras y fragmentos de ladrillo- para acomodar las piezas sobre la caja del canal. A este respecto debe tenerse en cuenta que, además de las posibles irregularidades de los elementos de la cubierta, el remate en media caña 


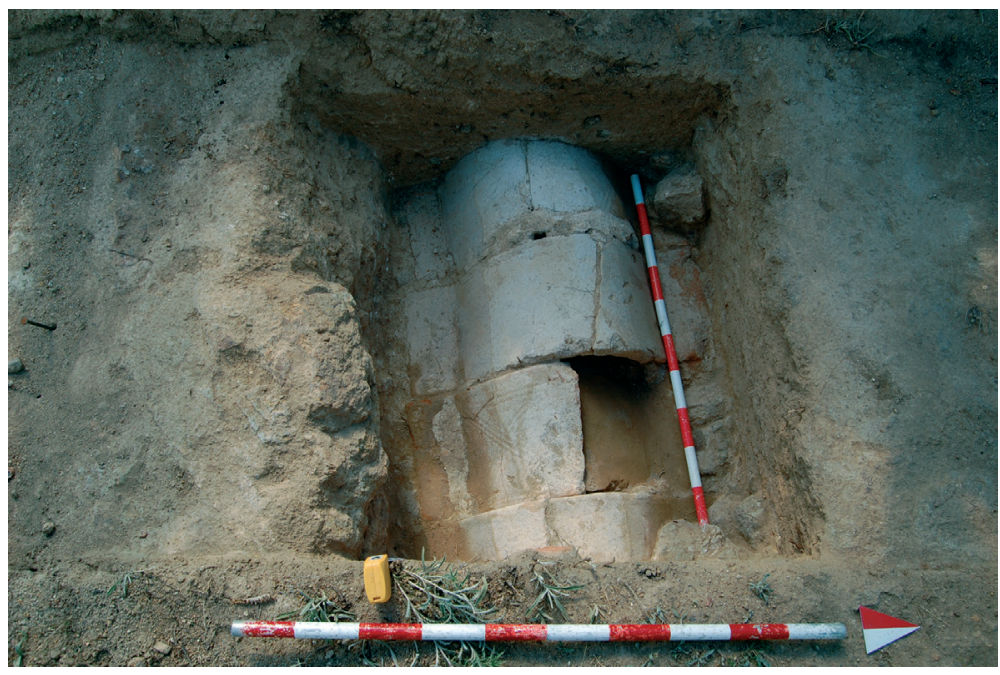

FIG. 5. Detalle de la cubierta de piezas cerámicas empleadas en la conducción.

el canal procedente de la Alcantarilla se cubrió con grandes losas rectangulares de granito, de aproximadamente $1,20 \mathrm{~m}$ de ancho, alrededor de un metro de longitud y unos $0,20 \mathrm{~cm}$ de espesor (Arenillas et al., 2009b: 154155). Las apreciaciones de estos autores eran, por tanto, correctas. El error en sus valoraciones procede, según se ha señalado, de la consideración de la existencia de un único abastecimiento a la ciudad, a partir de la unión teórica de dos tramos de ruinas de características diferentes. A este respecto cabe destacar que son numerosas, y no solo relativas a la cubierta, las diferencias tipológicas y de dimensiones entre el canal que parte de la presa y el excavado en los terrenos de la Academia, lo que permite confirmar que nos hallamos

que presenta el revestimiento de opus signinum fino del canal, sobre el que se apoyan los laterales de la bóveda, y las diferencias de altura entre los cajeros documentadas en algunos tramos dificultarían, sin duda, el correcto acoplamiento de estas piezas ${ }^{13}$.

El empleo de "losas de piedra o de barro cocido" como cubierta del sistema romano que abastecía Toledo desde la Alcantarilla ya fue señalado por Ortiz Dou (1948). También Aranda, Carrobles e Isabel (1997: 288, 291 y 294) destacan el uso de lajas de piedra para la cubierta del canal en el término de Mazarambroz y "numerosos fragmentos de losas de barro" junto al tramo de conducción de la ladera o del cerro de los Alcaravanes, ya en terrenos del campo de maniobras de la Academia. Y, efectivamente, tal y como hemos visto, la cubierta del acueducto excavado en el espacio militar está constituida por piezas de barro cocido. En contraposición,

13 Una cubierta de idénticas características fue documentada en el acueducto de Combes, en el abastecimiento de agua del establecimiento termal galo-romano de Aquae Nerii (Néris-Les-Bains, Francia) (Desnoyers, 1982 y 1983). De nuevo nos hallamos ante un sistema de abovedamiento mediante tejas curvas, de idéntica forma y dimensiones similares a las halladas en Toledo. Sin embargo, en el acueducto francés, estas piezas estaban protegidas por un solado de hormigón de unos $10 \mathrm{~cm}$ de espesor (Desnoyers, 1983: 76). El mismo autor hace referencia a un segundo acueducto en el que el sistema de cubierta empleado sería similar, el de Beaulieu, en la región del Loira (Desnoyers, 1983: 76). ante conducciones diferentes ${ }^{14}$.

En primer lugar, la altura de la caja del canal es notablemente mayor en el de la Alcantarilla -60 $\mathrm{cm}$ - que en el de la Academia $-32 \mathrm{~cm}-$. Tampoco la disposición y composición de los materiales que revisten la obra al interior son los mismos en ambos acueductos. Los muros cajeros de la conducción de la Alcantarilla se disponen directamente sobre un cimiento -substructio- más ancho -entre 7 y 12 $\mathrm{cm}$ por cada lado-, compuesto por un núcleo de opus caementicium entre alzados de mampostería. El interior de la caja del canal se reviste con un resistente mortero de arena fina que, desde la base, se prolonga perdiendo espesor por las caras internas de los cajeros. Cubriendo este primer nivel, y sólo en la parte inferior de la obra, se dispone una segunda capa, de 10-12 cm de grosor, rica en cal y con pequeños fragmentos de cuarzo de menos de $1 \mathrm{~cm}$, constituyendo un material muy resistente $\mathrm{e}$ impermeable. Finalmente, toda la caja del canal estaba protegida por una capa de opus signinum fino de unos $2 \mathrm{~cm}$ de espesor, que se prolongaría hasta rematar horizontalmente sobre la coronación de los cajeros. Este revestimiento formaba una escocia en los ángulos inferiores, donde se observa una mayor

14 Aunque el canal de la Alcantarilla no fue objeto de excavación, los datos recogidos en los numerosos tramos reconocidos permiten su caracterización con bastante definición (Arenillas et al., 2009b: 152). 


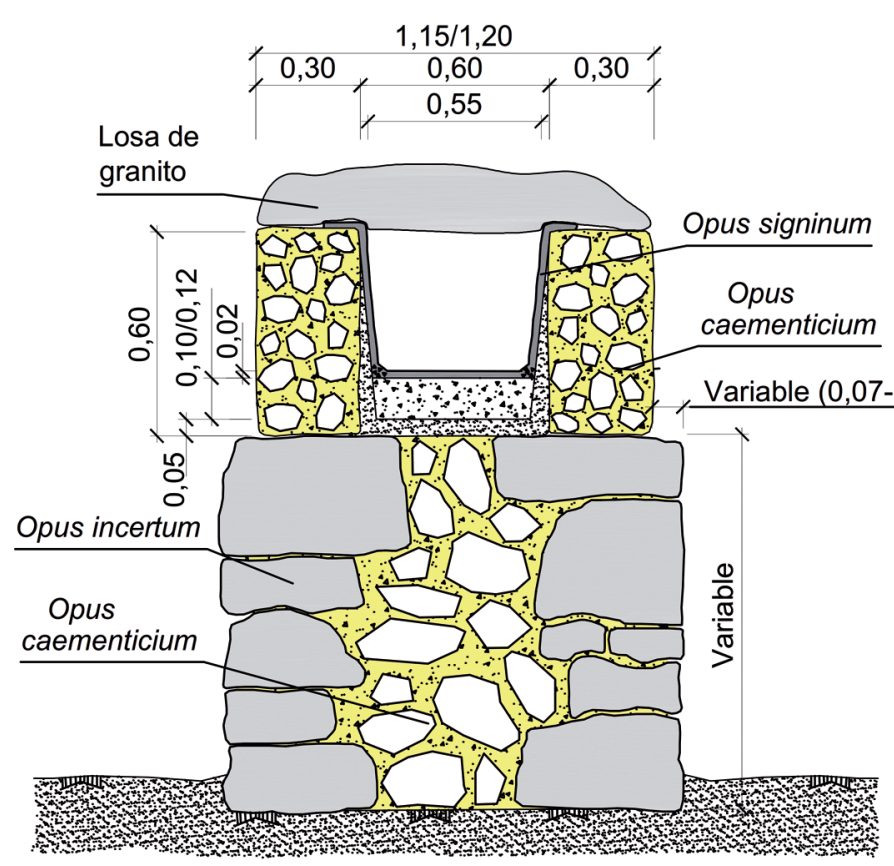

CANAL DE LA ALCANTARILLA: SECCIÓN TIPO

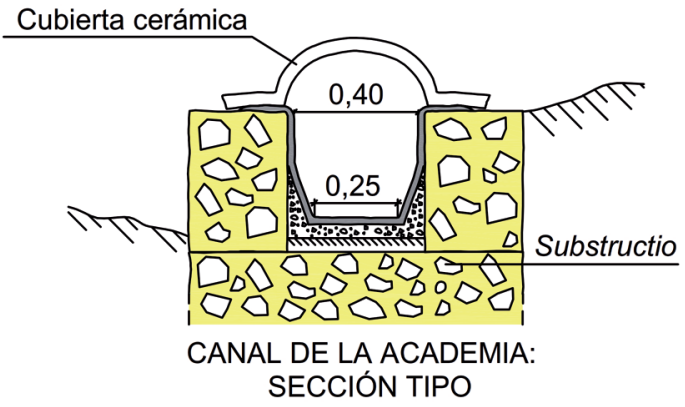

SECCIÓN TIPO

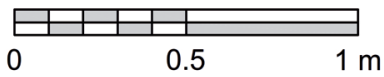

Fig. 6. Secciones comparadas del acueducto de La Alcantarilla y el excavado en los terrenos de la Academia.

dimensión en los fragmentos cerámicos machacados que lo componen, similar al opus signinum grosero existente en el canal de la Academia. Con estas características, la obra de la Alcantarilla contó con unas dimensiones interiores de $55 \mathrm{~cm}$ de ancho en la base y $60 \mathrm{~cm}$ en su parte superior, con una altura de unos 41-43 $\mathrm{cm}^{15}$ (Arenillas et al., 2009b: 151155), esto es, notablemente mayores que el canal excavado en esta intervención.

\subsection{Reparaciones y mantenimiento del acueducto}

No obstante, además de la bóveda cerámica descrita, se ha documentado en la ladera que desciende desde el cerro de los Alcaravanes hacia el arroyo de la Degollada -sondeo 6.24B- un tipo de cubierta diferente en el canal excavado, dispuesta

15 Lo que conduce a una superficie de la caja de $0,24 \mathrm{~m}^{2}$ frente a $0,10 \mathrm{~m}^{2}$ en el de la Academia. de forma adintelada y realizada con lajas de granito más o menos desbastadas con dimensiones de 55$60 \times 35 \mathrm{~cm}$ y un espesor medio de $15 \mathrm{~cm}$-muy diferentes, por tanto, a las del canal de la Alcantarilla-. Las piezas están trabadas con mortero de cal y se calzan con ripios de piedra o ladrillos fragmentadas, al igual que la bóveda cerámica documentada en la mayoría del recorrido de la conducción. Es posible que una cubierta similar, también de piedra, existiese a la altura del sondeo 6.9, puesto que se han documentado fragmentos de lajas de granito en su perfil.

Esta no es la única anomalía observada en la obra. En los sondeos 6.1в, 6.8 y 6.10 se documentó una variación del revestimiento que denominamos opus signinum fino, no superpuesto en ningún punto al anterior. Está realizado con trozos de ladrillo machacado de mayor tamaño, menor cantidad de cal y presenta, por tanto, un tono más oscuro, siendo además su acabado final más rugoso. $\mathrm{El}$ sondeo $6.1 \mathrm{~B}$ muestra igualmente otras reparaciones en la 


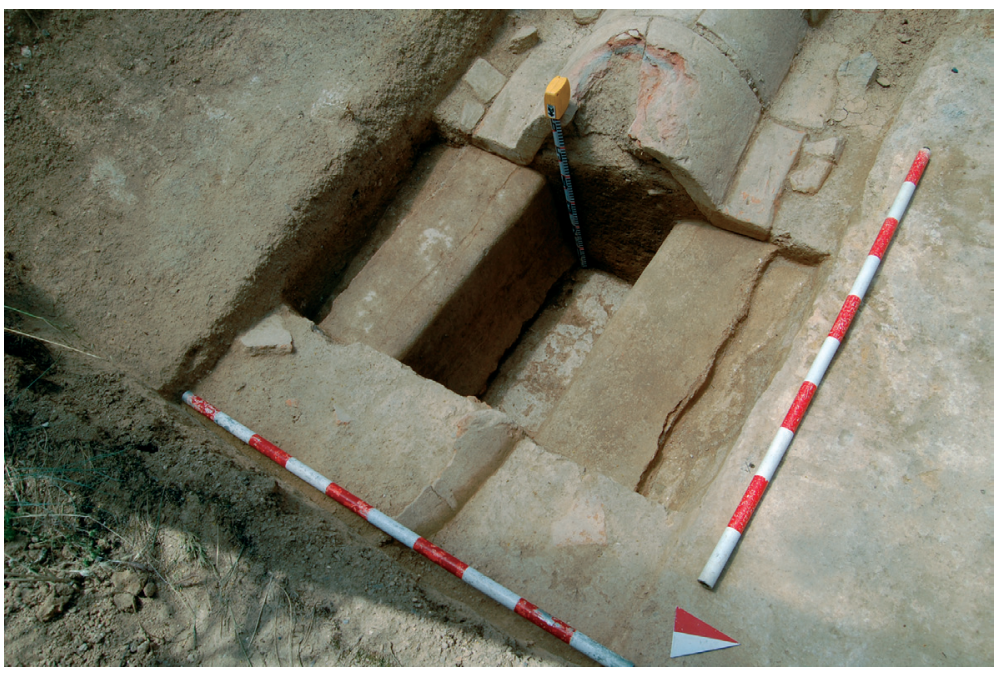

FIG. 7. Reparación en el revestimiento del canal documentada en el sondeo 6.21.

base del canal, realizadas estas sólo con mortero de cal. Por último, en el sondeo $9.1 \mathrm{~N}$, se documentó una tercera variante: también un fino enfoscado de mortero de cal, con apenas unos milímetros de espesor y aspecto rugoso. En este mismo tramo de la conducción, el preparado de piedras compactas de la base del canal sobre el que se dispone el opus signinum grosero tampoco existe, documentándose en su lugar una serie de fragmentos de teja, de 5 a $10 \mathrm{~cm}$ de tamaño medio, dispuestas de forma plana. Algo parecido cabe señalar en el sondeo 9.8 $\mathrm{N}$, donde el preparado de piedras de la base no presenta las mismas características documentadas en el resto de la obra, al estar realizado con elementos de mayor tamaño y formas más o menos planas, que se disponen también en horizontal.

Otra diferencia, observada en los sondeos 6.21, 12.1 y 12.3, es el remate del opus signinum fino en la coronación de los cajeros, que en estos sectores de la conducción no se realiza en media caña. En el caso del sondeo 6.21 sabemos que esta variación responde a una reparación en el canal, puesto que el opus signinum de los laterales de la caja presenta continuidad con el remate superior plano, pero no con el revestimiento de la base, sobre el que se apoya. Tampoco mantiene la forma achaflanada -en escocia- en la parte inferior de la conducción documentada en otros tramos.

Las diferencias señaladas en la cubierta y revestimientos del acueducto son, en nuestra opinión, el resultado de lógicas actuaciones de mantenimiento de una obra en uso probablemente durante un largo periodo de tiempo, puesto que las diferencias tipológicas observadas son notables en algunas ocasiones. Pese a que, como ya se señaló al inicio del artículo, la intervención por sondeos independientes merma la posibilidad de una secuenciación estratigráfica de las reparaciones, dada la inexistencia de relaciones directas entre los mismos, creemos que es posible establecer una posible secuencia constructiva en la obra a partir de los datos disponibles.

Debido al elevado número de veces en que se ha documentado y por su buena ejecución y factura, consideramos que la sección originaria del canal estaría revestida con un opus signinum fino, rematado en media caña sobre los cajeros y con una sección acabada en escocia en su parte inferior. Dadas las similitudes observadas entre el revestimiento de opus signinum fino considerado original y el documentado en el sondeo 6.21 que, sin embargo, remata en recto y no ofrece solución de continuidad con la base del canal, suponemos posible esta reparación en un segundo momento, probablemente cercano en el tiempo a la construcción originaria, puesto que la técnica y composición del revestimiento parecen idénticas. La misma intervención podría haber afectado a los tramos 12.1 y 12.3 del canal. En último lugar, aunque probablemente no coetáneos entre sí, situamos los revestimientos de opus signinum fino descritos con poca cantidad de cal, más oscuros o rugosos que el originario y las reparaciones con argamasa de cal observadas en el revestimiento del sondeo 6.1в. Consideramos además originaria la cubierta de piezas cerámicas -quizá perteneciente a distintos momentos dadas las diferencias en las pastas y cocciones observadas-, cuya restauración en momentos tardíos se llevaría a cabo por medio de grandes lajas de granito como las observadas en el sondeo 6.24B y, probablemente, en el 6.9.

Además de estas reparaciones vistas en la obra, indicativas de un largo periodo de uso, se ha documentado en numerosos sondeos $-6.1 \mathrm{~B}, 6.3,6.7$, $6.11,6.16,6.17,6.19$ y $6.21-$ un nivel de sedimento 
fino de espesor variable -entre 10 y $32 \mathrm{~cm}$ - que llega a colmatar el interior del canal. La existencia de este relleno nos indica que el acueducto continuó transportando agua una vez abandonado el sistema, hasta que los limos depositados acabaron colmatando en algunos puntos la conducción.

\section{La torre acuaria del Horno de Vidrio}

Dentro del recorrido del acueducto excavado, y formando parte del mismo, se encuentra la estructura de

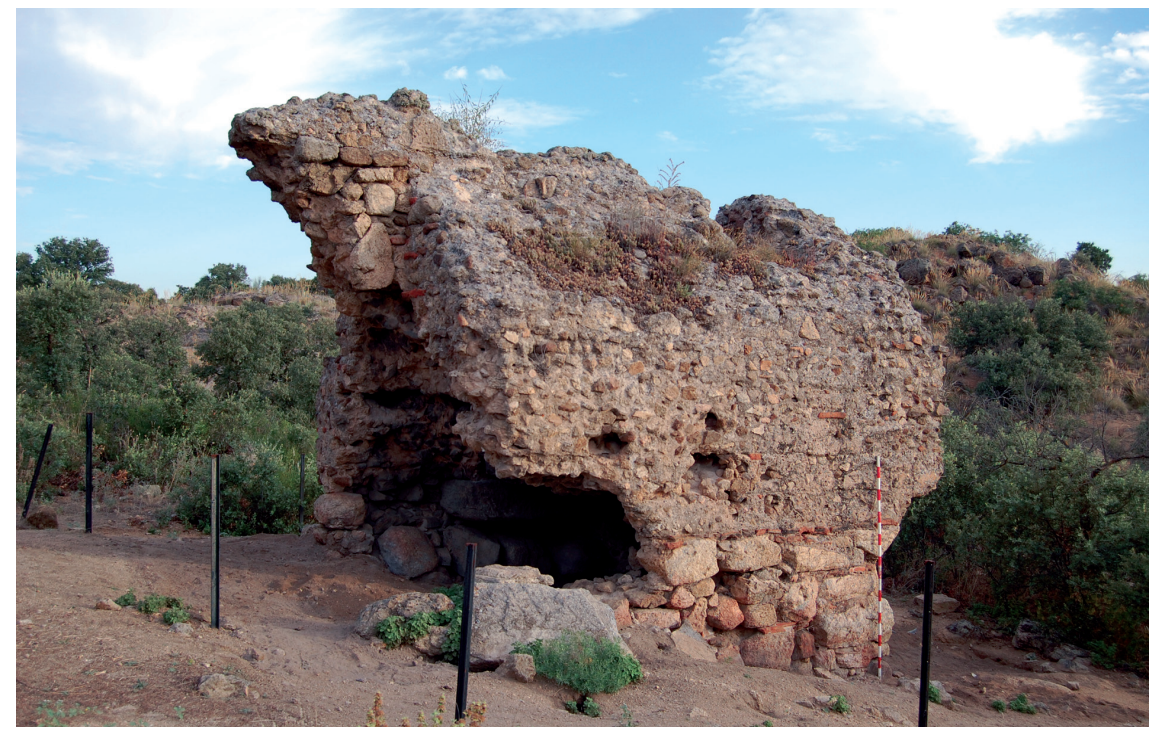

FIG. 8. Torre conocida como el Horno de Vidrio. pérdida de carga conocida como el "Horno de Vidrio". Se localiza en la margen meridional del Camino de la Sisla, en el lado o de la carretera que atraviesa de $s$ a $\mathrm{N}$ la mitad septentrional del campo de maniobras, en una ladera bastante pendiente. Está construida directamente sobre la roca del terreno -migmatita-, en la que se observan algunos cortes para acomodar su cimentación.

Se trata de una construcción muy sólida, aunque actualmente muy degradada, que forma un bloque muy compacto y casi macizo de 4,60 m de longitud, 3,70 $\mathrm{m}$ de ancho en su cara $\mathrm{N}$ y casi 3,90 en su lado s. Su altura ronda los $6 \mathrm{~m}$ siendo algo más elevado por el lado $\mathrm{s}-6,20 \mathrm{~m}$ frente a 5,80 m por la cara $\mathrm{N}-$. El material empleado para su construcción fue el hormigón -opus caementicium-, en el que se empleó material latericio como caementa. En el frente superior $\mathrm{N}$ de la torre, dos verdugadas de ladrillo fueron colocadas a modo de cornisa. Por último, la obra estaba revestida al exterior, en su base, con una fábrica de sillería irregular con numerosos ripios de piedra, ladrillo y tégulas.

El enlace del canal con la torre desde la ladera se conseguía mediante una estructura elevada que, al menos en su contacto con el Horno de Vidrio, remataba en un arco, siendo aún visible el arranque del mismo en el extremo $s$ de la construcción. La conducción discurría de $\mathrm{S}$ a $\mathrm{N}$ por el eje central de la

coronación de la torre, donde se conservan restos de la misma en su sector meridional, observándose aún parte del opus signinum grosero de su revestimiento. En el extremo N, un espacio hueco, vertical, de sección circular y unos $44 \mathrm{~cm}$ de diámetro recorre la estructura en toda su altura, permitiendo la caída del agua hasta el nivel inferior, en un salto de 5,5 $\mathrm{m}$. Dicho conducto conserva la mayor parte de las grandes piezas curvas de cerámica -dos por hiladaque lo forraban al interior. Estas son idénticas a las descritas para la cubierta de la conducción, sirviendo sus apoyos laterales como elemento de enjarje con la estructura de opus caementicium. La documentación del empleo de idénticos elementos en el canal excavado y en la torre del Horno de Vidrio -revestimiento de opus signinum grosero y piezas cerámicas características- apunta a que ambas construcciones formaron parte de una misma obra.

En la parte inferior de la cara $\mathrm{N}$ de la torre, $\mathrm{y}$ construida solidariamente con ella, encontramos una gran pila rectangular de granito con orientación $\mathrm{N}$-s, de más de 1,5 m de longitud y unos 0,64 $\mathrm{m}$ de ancho. En ella hay dos agujeros circulares, de unos $28 \mathrm{~cm}$ de diámetro cada uno, que fueron cegados con un relleno de piedras y mortero de cal probablemente desde su puesta en obra. La parte septentrional de la pieza se halla rota, observándose los restos de un tercer agujero de dimensiones 


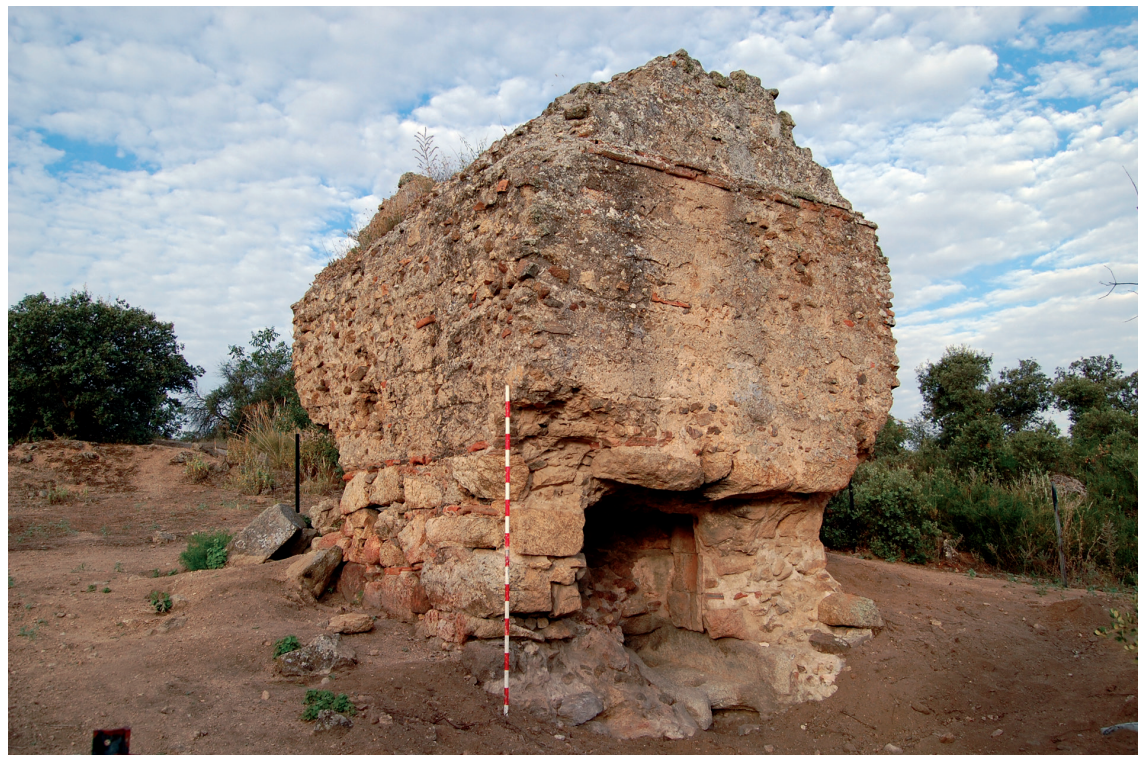

FIG. 9. Alzado norte del Horno de Vidrio.

un enorme hueco de unos 2 $\mathrm{m}$ de lado y 1,60 m de altura. La apreciación de restos de un revestimiento de opus signinum al que se adosa un gran bloque de opus caementicium, en la esquina superior o de este espacio, nos hizo suponer en un primer momento la posibilidad de una fase anterior en la obra, en la que habría podido existir un depósito de agua, quizá asociado a una fuente, dada la cercanía de esta estructura a la vía romana de Toledo a Córdoba -la vía xxx del It. Antonino-, que discurriría en paralelo al acueducto en este tramo (Arenillas et al., 2009b: 168169). La excavación de este

similares a los dos anteriores, por el que sí discurriría el agua. Existe además una muesca bastante profunda, de forma circular y labrada en la pared vertical del fondo de la pila. Ninguno de los agujeros descritos se corresponde claramente con la bajante vertical de la torre, lo que invita a pensar -junto a los conductos inutilizados- en una pieza reaprovechada. Bajo la pila de granito la excavación ha permitido documentar la existencia de una fosa excavada en la roca, que interpretamos como la zanja de construcción del canal de conducción. Dicha fosa está delimitada en su cara o por la prolongación de la fábrica de opus caementicium de la torre, que se extiende como un brazo hacia el $\mathrm{N}$, configurando el cajero izquierdo del acueducto. Las dimensiones de la zanja que albergaba la caja del canal son mayores bajo la pila de granito, $80 \mathrm{~cm}$ de ancho, que enseguida disminuyen hasta $60-70 \mathrm{~cm}$, por medio de un pequeño codo tallado en su lado derecho. Lo mismo ocurre con la cota del suelo, casi $20 \mathrm{~cm}$ más profunda bajo el salto de agua. Estas características parecen indicar la existencia de una pequeńa pileta de remansamiento de las aguas bajo la conducción vertical.

El sector meridional de la torre es la parte de más difícil interpretación, debido al fuerte expolio al que ha sido sometida, lo que ha generado espacio interior no pudo llevarse a cabo en su totalidad por miedo a dañar la torre. Sin embargo, fue posible documentar la cimentación de su muro de cierre meridional, además de una fosa tallada en la roca, cegada con piedras en un momento posterior y amortizada por la propia estructura del Horno de Vidrio. Su ubicación tampoco coincide con el eje actual de la conducción, sino que se halla ligeramente desviada hacia el o.

Pese a las incógnitas aún existentes sobre la interpretación de algunas partes de esta obra, la mayoría de los elementos descritos avalan su explicación tradicional como una construcción cuya función más evidente fue permitir una rápida pérdida de cota en la conducción. Esto supone además, como es obvio, la reducción de la velocidad del agua a la salida de la torre, ya que esta era muy elevada a la entrada, puesto que el canal discurre con fuerte pendiente en toda el área excavada, según veremos. Otro posible objetivo complementario de la estructura podría ser la retención de sedimentos en la pequeña pileta dispuesta al pie de la caída vertical, aunque sobre la función real de este tipo de elementos existe discusión (Chanson, 2000: 52). En cualquier caso, estos no fueron los únicos motivos para construir la torre. Su localización en el interfluvio entre los arroyos de la Degollada y la Rosa 
no es fortuita, y corresponde a una decisión muy clara y bien estudiada de sus constructores: facilitar el paso de la conducción de una cuenca a la otra, al tiempo que se conseguía la pérdida de cota antes indicada.

Debe también señalarse que el Horno de Vidrio es una construcción muy singular, no sólo dentro del sistema de abastecimiento de aguas a Toledo sino, en general, dentro de los dispositivos ideados por los ingenieros romanos para lograr la pérdida de carga en sus conducciones (Hodge, 1992: 160-161 y 2000b: 87-88; Chanson, 2000 y 2002: 8-10; Barahona 2014). La peculiaridad de esta estructura podría derivar de su

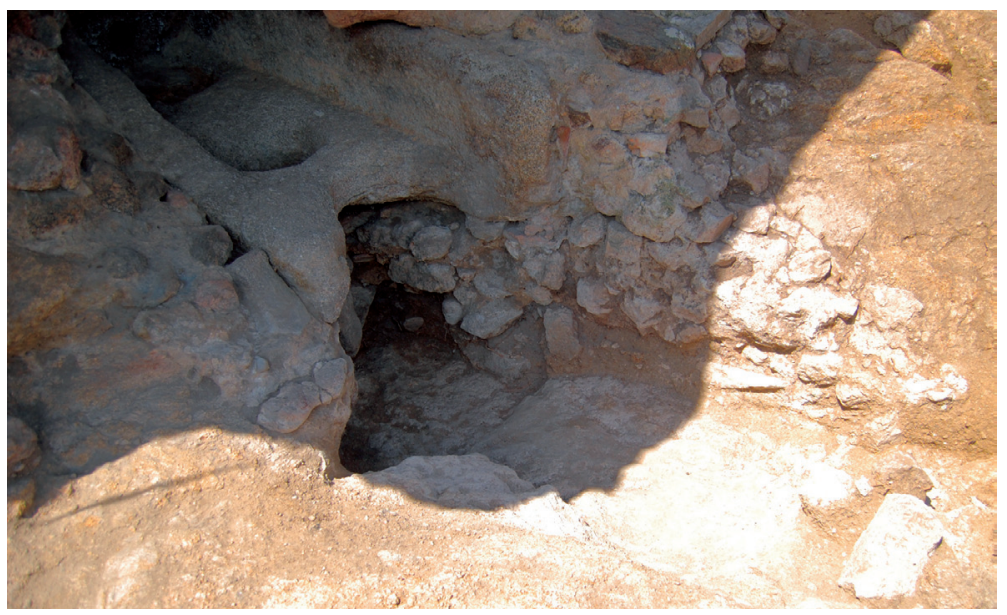

FIg. 10. Pileta excavada en la roca bajo el conducto vertical del Horno de Vidrio. construcción/reconstrucción a partir de una obra preexistente, ya que distintos elementos mencionados así lo avalarían. Tanto la gran losa de granito al pie de la cara $\mathrm{N}$ de la torre, como los ladrillos y tégulas reutilizados como ripios en su zócalo de cimentación o, más posiblemente, los restos del revoco de opus signinum en la pared interior o de la obra, evidencian el empleo de materiales que no fueron diseñados inicialmente con esa función, y que incluso pudieron traerse de otros lugares. En la misma línea, la fosa excavada en la roca en el interior s de la obra, detectada durante las excavaciones, podría haber formado parte de un elemento de pérdida de carga previo, lo que confirmaría esta hipótesis. Por último, como ya se ha señalado, las numerosas reparaciones documentadas a lo largo del recorrido del canal excavado indicarían un largo periodo de funcionamiento para este, por lo que no sería extraño pensar en la necesidad de reparar/reconstruir también otros elementos del sistema.

\section{Características técnicas de la conducción: pendiente y capacidad}

El recorrido del acueducto en los terrenos de la Academia se desarrolla, según se ha dicho, entre un punto situado en la margen derecha del arroyo de la Degollada y muy cercano a su cauce -sondeo 6.34- y otro localizado en la ladera $\mathrm{N}$ del Cerro

Cortado -sondeo 12.5-. La longitud total de la conducción entre ambos extremos -medida sobre un plano a escala 1: 2.000- es de unos $2500 \mathrm{~m}$, con una diferencia de cotas de $60 \mathrm{~m}$-cota 611 en el sondeo 6.34 y cota 551 en el $12.5-$, lo que determina para el canal -prescindiendo de los 5,5 $\mathrm{m}$ de pérdida de carga en el Horno de Vidrio- una pendiente media del 2,2\%, muy elevada para una conducción de estas características. Esta circunstancia se acusa aún más cuando se analiza el recorrido por sectores, evidenciándose tres tramos con distinta pendiente $^{16}$. El primero, de algo más de $400 \mathrm{~m}$, se desarrolla entre los sondeos 6.34 y 6.17 -cota 609-, este último en la ladera occidental del cerro de los Alcaravanes. En dicho recorrido la pendiente media resulta ser del $0,45 \%$, la menor de todo el trazado. El segundo está en prolongación del

16 Las conducciones romanas no mantenían una pendiente uniforme en todo su recorrido. Tampoco esta fue la pretensión de los ingenieros que las construyeron, que jugaban con ella para ahorrar altura en puentes y substructiones o reducir la longitud de los túneles. Además de ello, pudieron influir también en dichas variaciones posibles errores en las nivelaciones o la propia sistematización del trabajo para su construcción, con cuadrillas que trabajaban a la vez en diferentes sectores del acueducto, siempre desde aguas arriba hacia aguas abajo, lo que obligaba a ajustar los enlaces entre diferentes tramos (Grenier, 1960: 29; Mathews, 1970: 9; Hodge, 1992: 178-191; Ventura, 1993: 131). 
anterior y su longitud es algo menor de $600 \mathrm{~m}$, entre el citado sondeo 6.17 y la parte alta -entra$\mathrm{da}$ - de la torre del Horno de Vidrio - cota 594-. En él la pendiente media es muy fuerte $(3,2 \%)$. El tercero se prolonga desde la parte baja del Horno de Vidrio - cota 588- hasta el final del tramo excavado (sondeo 12.5, cota 551) con una longitud de unos $1,500 \mathrm{~m}$ y pendiente media del $2,4 \%$.

Son pocos los autores romanos que hacen recomendaciones sobre las pendientes de los canales: Plinio el Viejo $(H N 31,57)$ y, con anterioridad, Vitrubio (De arch. 8, 7), al que sigue Palladio ya en época tardía $(O p$. agr. 9, 11). El primero se limita a señalar que dicha pendiente no debe ser inferior al $0,02 \%-2 \times 10,000 \mathrm{~m}-$, cifra muy razonable y que, en esencia, está marcando un límite por debajo del cual pueden ser ya importantes los depósitos de eventuales sedimentos arrastrados por el agua ${ }^{17}$. Conviene además señalar que el límite marcado por Plinio está claramente en la línea de los criterios actuales de construcción de canales. Por su parte, las cifras que aparecen en el tratado de Vitrubio son notablemente más altas, 0,5\%, lo que algunos autores atribuyen a errores en la transcripción de las copias (Grenier, 1960: 29; Mathews, 1970: 9). En el caso de Palladio este error sería aún más evidente, ya que casi triplica la pendiente recomendada por Vitrubio. Los ejemplos bien conocidos de los acueductos de la ciudad de Roma o los de la de Lyon, en Francia, así como otros muchos citados por diversos autores mantienen sistemáticamente pendientes medias que solo en algunos casos superan el 0,3\% (Bonnin, 1984: 193 y 194; Hodge, 1992: 178-191 y 2000a: 50-53). En la Península Ibérica la tónica no es diferente (González Tascón y Velázquez, 2004: 112-113).

En comparación con estos ejemplos la pendiente del canal de la Academia destaca por sus valores muy elevados. En circunstancias como estas, los ingenieros romanos, conocedores de los efectos negativos que la velocidad del agua podía determinar en el revestimiento de los canales, emplearon diferentes dispositivos para reducir tales daños:

\footnotetext{
17 En sentido contrario, la velocidad del agua en los canales tampoco debe ser muy alta puesto que ocasionaría erosiones importantes en las conducciones. A este respecto cabe añadir que Bonnin (1984: 193) señala para las conducciones de Roma y en diversos acueductos de Francia y Austria, velocidades del agua que oscilaban entre 0,5 y $1 \mathrm{~m} / \mathrm{s}$.
}

rápidas, de corta longitud y fuerte pendiente; peldaños en las soleras y estructuras de pérdida de carga -normalmente pozos- (Hodge, 1992: 160-161 y 2000b: 87-88; Chanson, 2000 y 2002: 8-10). Las rápidas combinan cortos tramos de conducción con fuerte pendiente, con otros casi horizontales en los que suele ser necesario un ensanchamiento de la sección del canal o la reducción del resguardo - para mantener caudales- con el efecto de reducir la velocidad del agua ${ }^{18}$. En el caso de los peldañeados, estos suelen estar formados por una sucesión de escalones que determinan la pérdida de energía de la corriente y, en definitiva, la reducción de la velocidad del agua circulante. Los pozos de pérdida de carga son caídas verticales en las conducciones, normalmente empleadas en los tramos subterráneos. En ocasiones estos pozos pueden estar construidos sucesivamente, dispuestos en cascada. Aunque este tipo de dispositivos no son comunes en todos los acueductos, son relativamente numerosos los ejemplos documentados en Francia, Italia, Alemania, Argelia, Turquía (Hodge, 1992: 160-161 y 2000b: 87-88; Chanson, 2000: 47-52 y 2002: 8-10; González Tascón y Velázquez, 2004: 138-140) o la propia Península Ibérica (Ventura, 1993). Una variante de estos pozos, pero en superficie, sería el ejemplo de la torre del Horno de Vidrio, de la que no se ha encontrado paralelo en el mundo romano (Barahona, 2014).

En el caso que nos atañe y, según hemos visto, los ingenieros que diseñaron la conducción a Toledo construyeron, tras un primer tramo de pendiente relativamente reducida, otro en el que esta era mucho más elevada, cuyo elemento de parada era la propia torre de pérdida de carga del Horno de Vidrio que, a su vez, facilita el cambio de cuenca y, por tanto, un trazado más fácil del canal hacia Toledo. En cuanto al último tramo de la conducción, desde la base del Horno de Vidrio y hasta la ladera $\mathrm{N}$ del Cerro Cortado, se acepta que debía terminar en un depósito, del que no se han localizado restos, desde el cual partirían las tuberías del sifón necesario para cruzar el Tajo (Fernández Casado, 1983: 514-515; Aranda, Carrobles e Isabel, 1997: 314-330; Arenillas et al., 2009b: 170172). Sin embargo, dada la fuerte pendiente de la

18 En este sentido creemos que puede interpretarse la variación en la sección del canal entre $1 \mathrm{~m}$ y 1,20 m de ancho ya seńalada (ver epígrafe 2.1). 
conducción también en este último tramo -2,4\%-, este depósito debió tener necesariamente una doble finalidad, como elemento de parada de aguas, además de ser cabecera del sifón. En este sentido, Fernández Casado (1983: 535537) señala ejemplos similares en las conducciones de Tipasa o Leptis Magna en el N de África.

La pendiente excesiva de la conducción de la Academia puede tener una cierta justificación en los fuertes condicionantes topográficos del terreno por el que circula. En esta zona final de los barrancos de la Rosa y la Degolla$\mathrm{da}$, que desaguan directamente al Tajo, colector general del territorio en que nos encontramos, las pendientes de sus respectivos valles se incrementan de modo significativo a la vez que se estrechan. De este modo, un canal construido por estos ámbitos, al tenerse que ajustar casi obligatoriamente a la pendiente general del terreno, permite pocas opciones para su trazado. De aquí que la decisión de construir una conducción con las características vistas, esto es, muy pegada al terreno y de pendiente muy alta, hace suponer que sólo debió funcionar correctamente (es decir, sin continuas roturas) con bajos caudales. La sección y pendientes del tramo del acueducto excavado permiten la circulación de hasta 100 1/s -volumen limitado por la menor pendiente de todo su recorrido, es decir, la del primer tramo-, pero a costa de alcanzar velocidades superiores a los dos metros por segundo, muy altas y, por tanto, con consecuencias negativas muy acusadas -fuerte erosión y dańos significativos-. Debemos suponer entonces la circulación de un caudal menor por esta conducción.

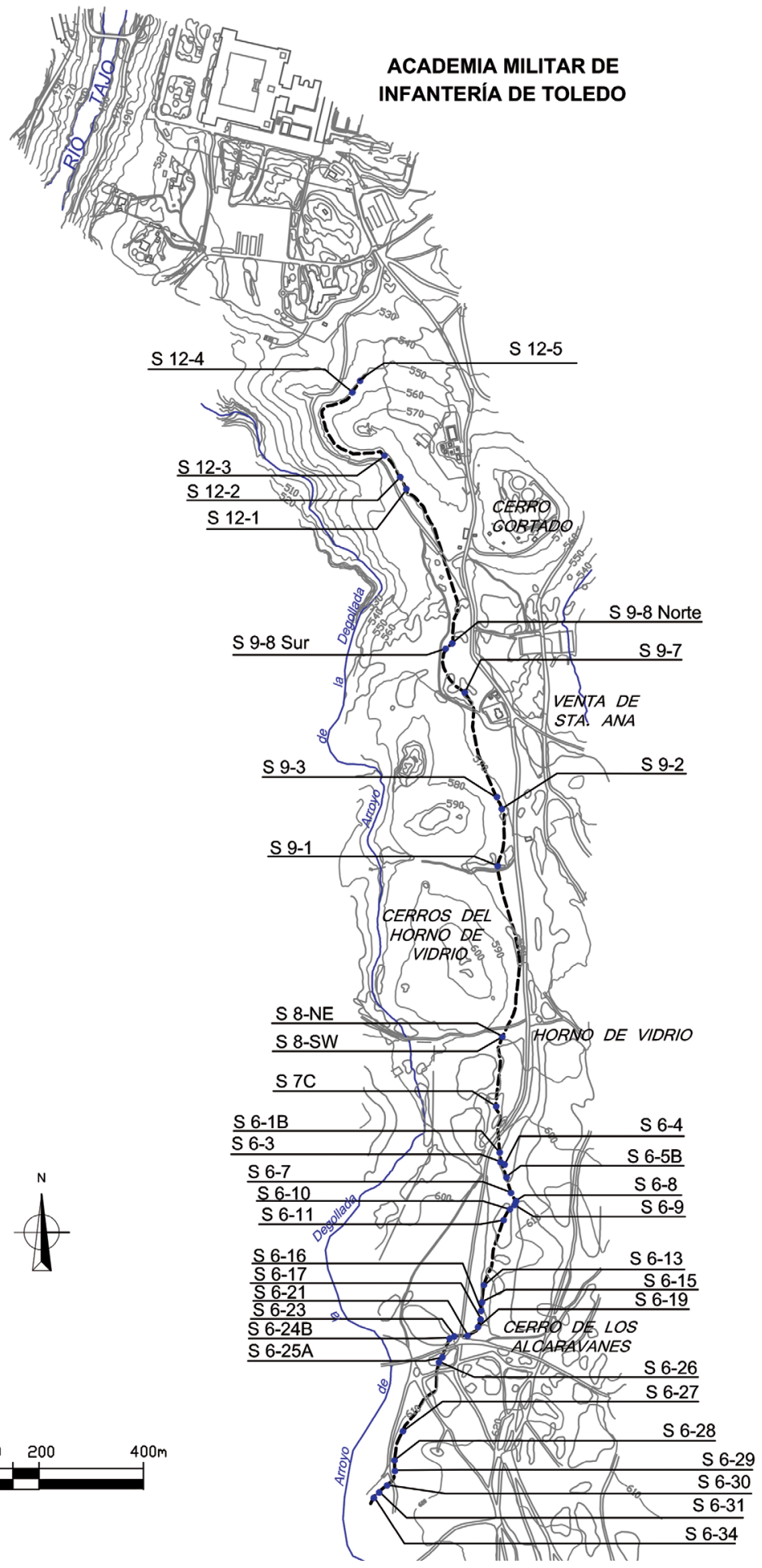

FIG. 11. Planta general del trazado del acueducto en los terrenos de la Academia. 


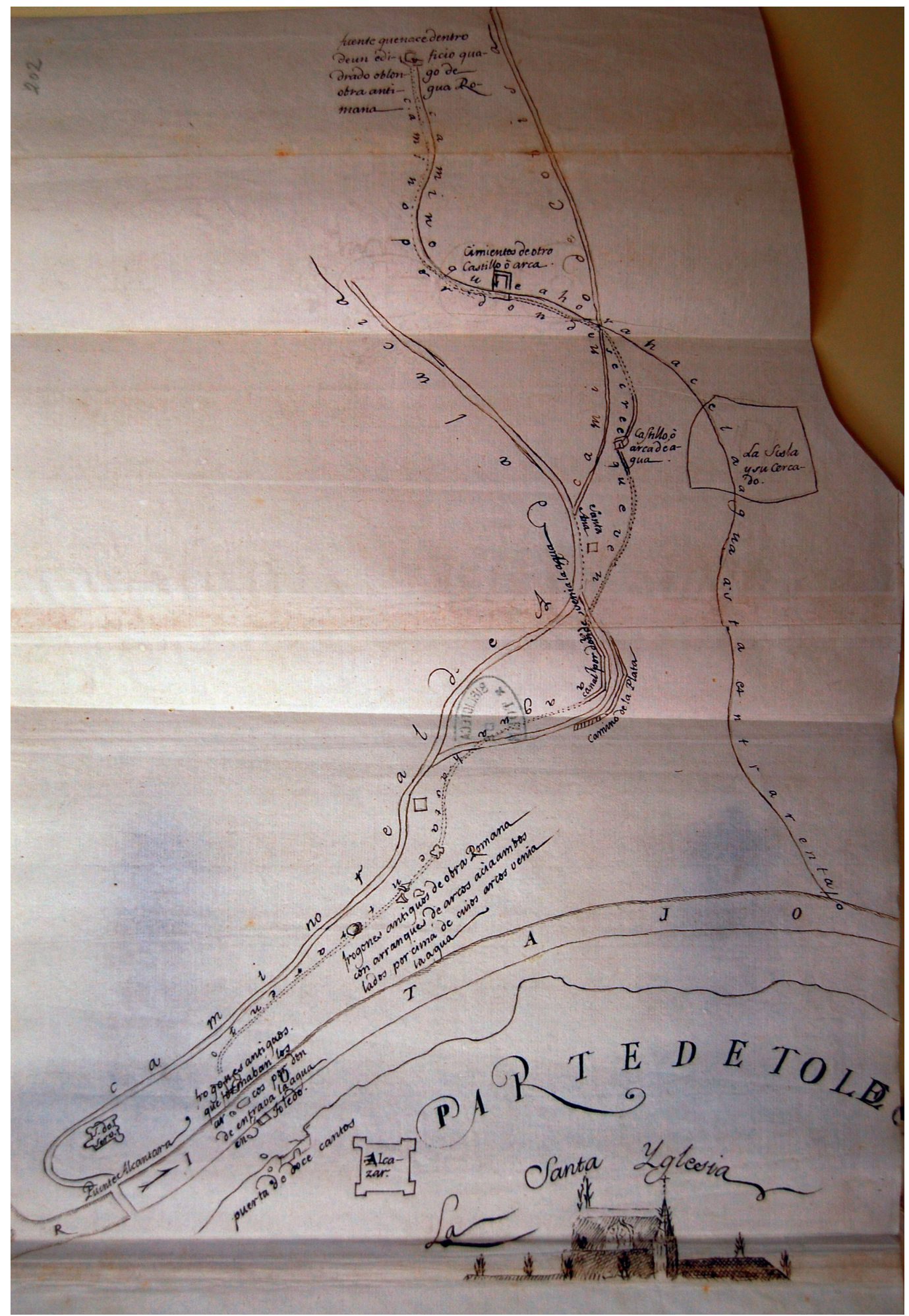

FIG. 12. Croquis con los restos del acueducto al SE de la ciudad de Toledo (Pérez Bayer, 1752). 


\section{El Camino de Cobisa, la calzada romana de Toledo a Córdoba y el trazado del acueducto en la antigua dehesa de la Sisla}

Además de los datos hasta ahora reseñados, la campaña arqueológica de excavaciones en la Academia Militar proporcionó también información relativa al trazado de dos caminos históricos del $s$ de Toledo y su relación diacrónica con el acueducto: el llamado Camino de Cobisa y la calzada romana de Toledo a Córdoba -la vía xxx del It. Antonino-.

$\mathrm{Al} s$ del tramo más meridional del canal localizado en esta campaña, y junto a la margen derecha del arroyo de la Degollada, se excavó parte de una instalación de época bajomedieval/moderna, quizá de carácter industrial y probablemente situada sobre el recorrido original de la conducción. En ella aparecieron fragmentos del revestimiento hidráulico del acueducto -opus signinum fino- en algunos niveles de excavación, así como formando parte de los mampuestos de uno de los muros. Inmediatamente al e de la instalación descrita pudo documentarse un antiguo camino empedrado, excavado parcialmente en el granito de la ladera. Evidencias de esta misma vía son visibles en dos sectores cercanos, a $40 \mathrm{~m}$ y $100 \mathrm{~m}$ al $\mathrm{N}$ del área excavada. En ambos sitios el camino se encuentra también parcialmente excavado en la roca y, el tramo más septentrional del mismo, corta además al antiguo canal romano entre los sondeos 6.31 y 6.34, por lo que cronológicamente es posterior a este. El recorrido que dibuja esta vía, actualmente desaparecida, aparece recogido en la primera edición del Mapa Topográfico Nacional, escala 1: 50.000 -hojas 629 y 657, 1882-. Este mismo trazado es recogido también por Pérez Bayer en un croquis donde dibujó las ruinas del acueducto en la dehesa de la Sisla, nombrándose la ruta como "Camino de Cobisa"19. Junto al mismo, el jesuita identifica la fuente caudalosa que considera origen del acueducto aunque, según parece deducirse de sus indicaciones, esta debió situarse más al $s$ del área excavada. Consideramos por tanto relevante la identificación de este antiguo camino de cara a futuros trabajos de investigación que permitan avanzar en el conocimiento de la red romana de abastecimiento a Toledo y, más concretamente, de los restos de la conducción que atraviesa los terrenos del campo militar.

19 вРт, 1752, ms. 128.
Una segunda intervención se llevó a cabo en el área conocida como el Olivar de Burguillos, junto al Camino Real de Toledo, identificado por Corchado y Soriano (1969: 137-138) como parte del trazado de una de las variantes de la calzada romana de Toledo a Córdoba -la vía xxx del It. Antonino-. Paralelo a esta ruta suponíamos que discurría el trazado del acueducto procedente de la cabecera del arroyo de la Rosa (Arenillas et al., 2009: 159 y 162). Sin embargo, la excavación llevada a cabo en esta área no proporcionó dato alguno que permita confirmar el paso por este punto de dicha conducción, tampoco el de la antigua calzada romana de Toledo a Córdoba. Los trabajos ejecutados pusieron al descubierto restos de muros de mampostería y tapial que relacionamos con una antigua linde. También parte de una antigua tubería de hierro de época reciente, probablemente perteneciente al abastecimiento de agua desde el arroyo de la Rosa a la ciudad de Toledo inaugurado en 1930, 'aguas de Burguillos', que estuvo en uso hasta la construcción del embalse del Guajaraz en 1971 (Barahona, 2009: 113-122). No es este el único punto donde queda en entredicho el recorrido tradicionalmente asignado a la calzada romana de Toledo a Córdoba. El antiguo camino empedrado que bordea las laderas $\mathrm{N}$ y E del Cerro Cortado, también identificado en la historiografía como parte de esta vía romana (Corchado y Soriano, 1969: 137-138; Ruiz Carmona, 2002: 59-60), presenta nuevamente incompatibilidad con el trazado del canal romano. Dicha imposibilidad fue ya apuntada en nuestro anterior estudio (Arenillas et al., 2009: 170), señalándose la necesidad de que la conducción tuviese, bien un paso superior bajo el cual discurriese la calzada, bien un paso subterráneo bajo la vía. Los resultados de los presentes trabajos descartan ambas opciones, dada la cota a la que discurría el acueducto en este punto -aproximadamente $1 \mathrm{~m}$ sobre el camino-, lo que impediría su tránsito. Ello supone que el camino que rodea Cerro Cortado o, más concretamente, la parte más meridional del trazado conservado, es posterior a la construcción y abandono de la conducción romana.

\section{Consideraciones finales}

La intervención realizada ha permitido conocer con alto grado de detalle el recorrido, forma constructiva y detalles técnicos del tramo final de uno de 
los sistemas que abastecieron a la urbe de Toledo en época romana, así como otros datos sobre su mantenimiento -reparaciones- y paulatino abandono. Las características de la obra permiten afirmar que nos hallamos ante los restos de una conducción de importancia, muy diferente, no obstante, al acueducto de la Alcantarilla, lo que ratifica la existencia de una red compleja de abastecimiento a la ciudad.

Respecto a su origen -caput aquae-, la pendiente del primer tramo del canal y su recorrido parecen señalar que este procede de un punto concreto del valle del arroyo de la Degollada, muy próximo al cauce. Según nuestra propuesta original (Arenillas et al., 2009b: 158-172), tras la unión de los sistemas de la Alcantarilla y de la Rosa a la altura de la población de Burguillos, el trazado de ambas conducciones discurriría a lo largo del interfluvio de los arroyos de la Rosa y la Degollada hacia Toledo, aunque los últimos kilómetros de este supuesto recorrido no pudieron ser adecuadamente contrastados en aquel momento. El resultado negativo de los sondeos realizados en esta intervención en el área del Olivar de Burguillos y el trazado claramente orientado hacia la Degollada de los restos del canal excavado permite plantear si el trazado en túnel de la conducción conjunta de la Rosa-la Alcantarilla, una vez dentro de los terrenos de la Academia, podría haber discurrido más hacia el o, por la margen derecha del arroyo de la Degollada, donde continuaría su recorrido a cielo abierto. En tal caso, los restos de la conducción documentada podrían pertenecer al sistema de la Rosa. A este respecto hemos de recordar que, tanto el jesuita Francisco Pérez Bayer ${ }^{20}$ como el historiador toledano A. Martín Gamero o R. Amador de los Ríos hablan del "alumbramiento" de agua en la dehesa de la Sisla, en una zona próxima al monasterio. Así, según se detalló ya en páginas anteriores, Pérez Bayer hace referencia a unas ruinas de las que mana el agua a "borbollones", situadas a unos 600 pasos de la Sisla, junto al Camino de Cobisa, de donde supone tomaría agua el acueducto. Por su parte Martín Gamero ([1862] 1979: 175) indica que "surtían los romanos a la ciudad de aguas potables, no tomadas del Tajo, sino traídas desde las vertientes de la dehesa titulada de San Martín de la Montiña, del Castañar y el puerto de Yébenes, y recogidas y alumbradas ${ }^{21}$ en el sitio llamado Viña Vieja por cima de la Sisla”. Si de estos pretendidos orígenes

20 BPT, 1752, ms. 128.

${ }^{21}$ El subrayado es nuestro. se suprimen Los Yébenes, por cuestiones topográficas evidentes, y las aguas de San Martín de la Montińa, cuya desvinculación con el sistema de abastecimiento a Toledo ya se explicó, quedan el Castañar, donde nace el río Guajaraz, en el que se sitúa la presa de la Alcantarilla, y la Viña Vieja, que podría coincidir con el origen fijado por Pérez Bayer. Por último, Rodrigo Amador de los Ríos (1905: 23) indica que el acueducto romano traía las aguas a Toledo de los manantiales ya citados por Martín Gamero, así "como de otros varios, desde cerca de Burguillos, por la torre acuaria, llamada Horno de Vidrio", pudiendo estar apuntando quizá, además, a la captación en el arroyo de la Rosa. En definitiva, consideramos factible que estas referencias documentales al "alumbramiento" de aguas al s del monasterio de la Sisla pudieran estar indicando la salida de una conducción en galería a la superficie, máxime cuando las características geológicas del valle de la Degollada no permiten la existencia de ninguna fuente de cierta entidad en este entorno. Este punto, si seguimos las indicaciones proporcionadas por Pérez Bayer, no puede estar lejos del lugar topográficamente más alejado de Toledo que se ha alcanzado durante la presente campaña, sino aguas arriba. De momento, sin embargo, y en tanto no se realicen nuevas excavaciones, debemos tomar como hipótesis esta propuesta.

Por otra parte, no puede descartarse tampoco completamente una posible toma del acueducto en el propio arroyo de la Degollada. Sin embargo, a este respecto cabe decir que la capacidad del canal excavado en la Academia, del orden de los 100 1/s según se ha dicho, es muy superior, sin lugar a dudas, al caudal que podría proporcionar este arroyo, pero perfectamente compatible con las aportaciones medias estimadas para las conducciones de la Rosa $-25 \mathrm{l} / \mathrm{s}-$ y la Alcantarilla -100 1/s- ${ }^{22}$ (Arenillas, 2009: 196-197).

En cuanto al destino de las aguas, parece claro que la obra excavada se construyó para el abastecimiento del cerro sobre el que se elevaba la ciudad romana de Toletum. La importante capacidad de la estructura, su cercanía a la urbe y la vinculación con el puente-sifón -venter- sobre el río Tajo son tres

22 Máxime teniendo en cuenta la más que probable posibilidad de que este último acueducto abasteciese también a su paso, al menos a la villa romana de Layos y, probablemente, a otras localizaciones a lo largo de su recorrido, por lo que el caudal que llegase a las cercanías de la ciudad sería menor (Arenillas et al., 2009b: 197-198). 
argumentos que permiten llevar a cabo esta afirmación. Algunos autores (Aranda, Carrobles e Isabel, 1997: 69 y 318) han indicado también la posibilidad de que parte de estos caudales se derivaran antes del paso del Tajo para su uso en la cercana zona de Cabrahigos, donde han aparecido restos de un complejo termal de gran entidad (Rojas, 1996).

En cuanto a la cronología de la obra, sus características constructivas, así como algunos de los materiales cerámicos aparecidos en contextos de amortización del canal o empleados como caementa en su construcción, remiten a un horizonte romano. En la excavación del acueducto destaca la aparición de un pequeño fragmento de Terra Sigillata Hispánica en el sondeo 6.8 y otro de cerámica común a torno con engobe rojo, en el sondeo 6.13. También un borde de cerámica común a torno con restos de opus signinum en el sondeo 6.9, probablemente embutido en origen en la fábrica del canal, al igual que otros fragmentos encontrados en los sondeos 6.1в, 6.8 y 6.11. El resto de material aparecido en la excavación de la conducción se limita a restos de cerámica común a torno de pastas anaranjadas y desgrasantes finos y un fragmento de cerámica común a torno de pasta amarillenta con acanaladura, localizado en el sondeo 12.1. En el Horno de Vidrio los materiales recuperados no aparecen en niveles de fundación, destacando fragmentos de cerámica común a torno, cerámicas vidriadas de cronología medieval/moderna, lozas, munición, fragmentos de metralla de época reciente y cuatro monedas de los periodos bajomedieval y moderno.

La posibilidad de establecer paralelos tipológicos a partir de los singulares elementos de la cubierta del canal tampoco aporta por el momento mucha información para datar la obra en los terrenos de la Academia. Sabemos que el acueducto de Aquae Nerii quedó amortizado a principios del s. III d. C., estimándose su construcción en la segunda mitad del s. I d. C. (Desnoyers, 1982: 145-147 y 1983: 77). Del de Beaulieu, como ya se ha señalado, apenas contamos con la referencia de su existencia.

No poseemos, por tanto, por el momento, datos directos suficientes que permitan acotar la datación del acueducto estudiado con mayor precisión que la de época romana. Sin embargo, la información que manejamos tampoco contradice la tradicional vinculación de la construcción del sistema hidráulico de abastecimiento a la ciudad con la obtención del rango de municipalidad de Toletum a lo largo del s. I d. C., ya señalada (Aranda, Carrobles e Isabel, 1997: 334-336; Rubio, 2005; Cauce, 2009). Según se indicó en apartados anteriores, la mayoría de estructuras relacionadas con el almacenamiento y distribución de agua excavadas en el interior del cerro toledano fueron construidas a lo largo del s. I d. C., por lo que hemos de suponer que en esas mismas fechas debía estar construido al menos uno de los acueductos. La cercanía a la ciudad y su vinculación directa con el puente-sifón sobre el Tajo hacen del canal de la Academia la opción más probable. A este primer sistema se incorporarían con posterioridad el de la Alcantarilla y, quizá, el de la Rosa -si es que no se trata de la misma obra-. En cuanto al momento de su amortización, de nuevo hemos de remitir a los datos de la red de distribución del interior de la ciudad, que dejó de funcionar a lo largo del s. Iv d. C. Sin embargo, en este caso, la existencia de más de un punto de suministro de agua evidencia un panorama más complejo para el que por el momento no tenemos respuesta.

\section{Bibliografía}

AMAdOR DE los Ríos, R. (1905): Monumentos arquitectónicos de España. Toledo. Madrid: edit. Martín y Gamaneta.

Aranda, F.; Carrobles, J. e Isabel, J. L. (1997): El sistema hidráulico romano de abastecimiento a Toledo. Toledo: Instituto Prov. de Investigaciones y Estudios Toledanos.

Arenillas, L.; Arenillas, M.; Díaz-Guerra, C. y MaCÍAs, J. M. (1999): "El abastecimiento de agua a Toledo en época romana”. En Macías, J. M. y Segura, C. (coords.): Historia del abastecimiento y usos del agua en la ciudad de Toledo. Madrid: Confederación Hidrográfica del Tajo, pp. 35-48.

Arenillas, M. (2009): "Conclusiones". En El abastecimiento de agua a Toledo en época romana. Madrid: Confederación Hidrográfica del Tajo, pp. 189-207.

Arenillas, M.; Barahona, M.; Gutiérrez, F. y Cauce, C. (2009a): El abastecimiento de agua a Toledo en época romana. Madrid: Confederación Hidrográfica del Tajo.

Arenillas, M.; Barahona, M.; Gutiérrez, F. y Cauce, C. (2009b): "Los sistemas de abastecimiento de agua a Toledo en época romana”. En El abastecimiento de agua a Toledo en época romana. Madrid: Confederación Hidrográfica del Tajo, pp. 123-185.

Arribas, R. (2008): "Nuevas aportaciones para el estudio del uso y distribución del agua en la ciudad romana de Toletum". En IV Congreso de las Obras Públicas en la 
ciudad romana (Lugo, Guitiriz, 2008). Madrid: CITOP, pp. 263-273.

Barahona, M. (2009): "Análisis documental". En El abastecimiento de agua a Toledo en época romana. Madrid: Confederación Hidrográfica del Tajo, pp. 61-122.

Barahona, M. (2014): “The Glass Kiln (El 'Horno de Vidrio'), a pressure drop tower in the water supply to the city of Toledo (Spain) during the Roman Era”. En Ohlig, Ch. \& Tsuk, T.: Cura Aquarum in Israel II. Water in Antiquity. Proceedings of the 15th International Conference on the History of Water Management and Hydraulic Engineering in the Mediterranean Region (Israel, 2012). Siegburg: Deutschen Wasserhistorischen Gesellschaft, pp. 265-273.

Barahona, M.; Arenillas, M.; Gutiérrez, F.; Martín, M.; Gómez, J. C. y García, F. (2007): “Un nuevo sistema de abastecimiento de agua a Toledo en época romana: La galería de la Pozuela”. En Actas del Quinto Congreso Nacional de Historia de la Construcción (Burgos, junio 2007). Madrid: Centro de Estudios Históricos de Obras Públicas y Urbanismo, pp. 1-10 (http:// www.sedhc.es/biblioteca/actas/CNHC5_008-M.\%20 Barahona.pdf).

Blázquez, J. M. (2011): "Los acueductos romanos en Hispania”. En Lagóstena, L. G.; CañIzar, J. C. y Pons, L. (eds.): AQVAM PERDVQUENDAM CURAVIT. Captación, uso y administración del agua en las ciudades de la Bética y el occidente romano. Cádiz: Seminario Agustín de Horozco de Estudios Económicos de Historia Antigua y Medieval. Universidad de Cádiz, pp. 95-126.

Bonnin, J. (1984): L'eau dans l'antiqué. L'hydraulique avant notre ère. Paris: Eyrolles.

Cauce, C. (2009): "Apuntes sobre la ciudad de Toledo en época romana”. En El abastecimiento de agua a Toledo en época romana. Madrid: Confederación Hidrográfica del Tajo, pp. 27-35.

Celestino, R. (i976): "El pantano romano de Alcantarilla, en Mazarambroz", Toletum, 7, pp. 161-178.

Chanson, H. (2000): "Hydraulics of Roman Aqueducts: Steep Chutes, Cascades and Dropshafts", American Journal of Archaeology, 104, 1, pp. 47-72 (http://espace.library. uq.edu.au/eserv.php?pid=UQ:9345\&dsID=PAP_96E.pdf). http://dx.doi.org/10.2307/506792

Chanson, H. (2002): "Certains aspects de la conception hydraulique des aqueducs romains", La Houille Blanche, 6/7, pp. 1-16.

Corchado y Soriano, M. (1969): "Estudio sobre vías romanas entre el Tajo y el Guadalquivir”, Archivo Español de Arqueología, 42, pp. 124-158.

Desnoyers, M. (1982): "Néris-Les-Bains (Allier), Ville thermale galo romaine", Revue archéologique du centre de la France, 21, 2, pp. 145-168.

http://dx.doi.org/10.3406/racf.1982.2348
Desnoyers, M. (1983): "Nouvelles recherches sur les aqueducs de Néris-les-Bains (Allier)". En Boucher, J. P. (dir.): Journées d'études sur les aqueducs romains (Lyon, 1977). Paris: Les Belles Lettres. Études Anciennes, pp. 75-80.

Fernández Casado, C. (I96I): "Las presas romanas en España”, Revista de Obras Públicas, 109, pp. 357-363. (http://ropdigital.ciccp.es/pdf/publico/1961/1961_tomoI_2954_06.pdf).

Fernández Casado, C. (I972): Acueductos romanos en España. Madrid: IETCC.

Fernández Casado, C. (1977a): "Los depósitos de agua de las conducciones romanas", Revista de Obras Públicas, 124, pp. 379-398. (http://ropdigital.ciccp.es/pdf/ publico/1977/1977_mayo_3145_01.pdf).

Fernández Casado, C. (1977b): “Contestación del autor a los comentarios publicados en nuestro número anterior sobre el artículo 'Los depósitos de agua de las conducciones romanas", Revista de Obras Públicas, 124, pp. 809-812 (http://ropdigital.ciccp.es/pdf/publico/1977/1977_octubre_3150_05.pdf).

Fernández Casado, C. (I983): Ingeniería hidráulica romana. Madrid: Turner, D.L.

Fernández Miranda, M.; Mangas, J. y Plácido, D. (I 990): "Indigenismo y romanización en la cuenca media del Tajo. Planteamiento de un programa de trabajo y primeros resultados". En Actas del I Congreso de Arqueología de la provincia de Toledo. Toledo: Diput. Provincial. Servicio de Arqueología, D.L., pp. 13-65.

GarcíA-Diego, J. A. (1974): "La cueva de Hércules", Revista de Obras Públicas, 121, pp. 683-700 (http:// ropdigital.ciccp.es/pdf/publico/1974/1974_octubre_3114_01.pdf).

García-Diego, J. A. (1975): “Contestación de José Antonio García-Diego a los 'Comentarios al artículo «La Cueva de Hércules»...”, Revista de Obras Públicas, 122, pp. 338-342 (http://ropdigital.ciccp.es/pdf/publico/1975/1975_mayo_3121_06.pdf).

García-Diego, J. A. y Porres, J. (1977): “Comentario al artículo 'Los depósitos de agua de las conducciones romanas', de Carlos Fernández Casado, publicado en Mayo de 1977", Revista de Obras Públicas, 124, pp. 727-730 (http://ropdigital.ciccp.es/pdf/publico/ 1977/1977_septiembre_3149_06.pdf).

González Tascón, I. y Velázquez, I. (2004): Ingeniería romana en Hispania. Historia y técnicas constructivas. Madrid: Fundación Juanelo Turriano.

Grenier, A. (1960): Manuel d'archéologie gallo-romaine. Les monuments des eaux. Paris: A. et J. Picard et Cie., vol. IV.

Hodge, A. T. (1992): Roman Aqueducts \& Water Supply. London: Duckworth.

Hodge, A. T. (2000a): "Aqueducts". En Wikander, Ö. (ed.): Handbook of Ancient Water Technology. Leiden, Boston, Köln: Brill, pp. 39-65. 
Hodge, A. T. (2000b): "Engineering Works". En WikanDER, Ö. (ed.): Handbook of Ancient Water Technology. Leiden, Boston, Köln: Brill, pp. 67-94.

Jiménez, A. (1976): "Los acueductos de Emerita". En Augusta Emerita. Actas del Simposio Internacional Conmemorativo del Bimilenario de Mérida (noviembre 1975). Madrid: Dirección General del Patrimonio Artístico y Cultural del Ministerio de Educación y Ciencia y Patronato de la ciudad de Mérida, pp. 111-125.

Lavado, P. J. (1988): "La mezquita del Solarejo o de Tornerías en Toledo". En I Congreso de Historia de Castilla la Mancha (Ciudad Real, 1985). Toledo: Junta de CastillaLa Mancha, D.L., vol. v, pp. 33-37.

Lavado, P. J. y Martín, P. (1986): "Excavaciones en la mezquita de las Tornerías (Toledo)”. En Actas del I Congreso de arqueología medieval española. Zaragoza: Diput. General de Aragón, Departamento de Cultura y Educación, D.L., vol. III, pp. 493-503.

López de Ayala, J. [1905] (1959): Catálogo monumental de la provincia de Toledo. Toledo: Diput. Provincial.

Martín Gamero, A. [1862] (1979): Historia de la ciudad de Toledo. Toledo: Zocodover, vol. I.

Matthews, K. D. (1970): "Roman aqueducts. Technical aspects of their constructions", Expedition: The magazine of the University of Pennsylvania, 13 (1), pp. 2-16.

Ortiz Dou, A. (1947): "Abastecimiento de agua potable a Toledo", Revista de Obras Públicas, 95, pp. 285-291 (http://ropdigital.ciccp.es/pdf/publico/1947/1947_tomoI_2787_01.pdf).

Ortiz Dou, A. (1948): Aguas de Toledo. Madrid: Mop. Servicios Hidráulicos del Tajo.

Porres, J. (1970): "El abastecimiento romano de aguas a Toledo". En Comunicación a la IV Asamblea de Instituciones de Cultura de las Diputaciones. Toledo: Diput. Provincial de Toledo.

Porres, J. (1975): “Comentarios al artículo 'La Cueva de Hércules', de José Antonio García-Diego, publicado en el mes de Octubre de 1974", Revista de Obras Públicas, 122, pp. 333-338 (http://ropdigital.ciccp.es/pdf/publico/1975/1975_mayo_3121_05.pdf).

Porres, J. (1983): "La mezquita toledana del Solarejo, llamada de las Tornerías”, Al-Qantara, Iv, pp. 411-421.

Porres, J. (1984): "Reconocimiento del acueducto romano en 1753 por Burriel y Palomares", Toletum, 14, 105-110.

Ponz, A. [1787] (1972): Viage de España, en que se da noticia de las cosas más apreciables, y dignas de saberse, que hay en ella. Madrid: Atlas, vol. I.

Prieto, G. (1990): "Santa María la Blanca y la mezquita de Tornerías: dos excavaciones de urgencia en Toledo". En Actas del I Congreso de Arqueología de la provincia de Toledo. Toledo: Diput. Provincial. Servicio de Arqueología, D.L., pp. 459-481.
Rey, A. (1928): "Bosquejo geomorfológico del peñón toledano", Boletín de la Real Academia de Bellas Artes y Ciencias Históricas de Toledo, 36-37, pp. 148-176.

Rojas, J. M. (1996): "Paseo de la Rosa, 76 (La piscina romana de Cabrahigos)". En Sánchez-Palencia, F. J. (coord.): Toledo. Arqueología en la ciudad. Toledo: Junta de Comunidades de Castilla-La Mancha, pp. 67-81.

Rojas, J. M.; Gómez, A.; Pérez , J.; Garrido, G. y Pereira, J. (2007): "El convento de Madre de Dios: Evolución histórica de una manzana de Toledo a través de la arqueología”. En PAssini, J. e IzQuierdo, R. (coords.): La ciudad medieval de Toledo: historia, arqueología y rehabilitación de la casa: el edificio Madre de Dios, 2005. Cuenca: Univ. de Castilla-La Mancha, 281-319.

Rubio, R. (2005): "Toledo romano: balance y nuevas perspectivas en la investigación”. En SÁnchez-Chiquito, S. (coord.): Arqueología romana en Toletum: 19852004. Puesta en valor de las termas romanas de la plaza de Amador de los Ríos. Toledo: Consorcio de la ciudad de Toledo, pp. 135-142.

Ruiz Carmona, S. (2002): Los caminos medievales de la provincia de Toledo. Análisis arqueológico e interpretación histórica. Madrid: Archiviana.

Ruiz Taboada, A. y Arribas, R. (2007): “El acceso Norte a la Toletum romana: el descubrimiento de una vía monumental bajo el conjunto del Cristo de la Luz", El Nuevo Miliario, 4, 5-13.

SÁenz, F. (1977): "Comentario al artículo 'Los depósitos de agua de las conducciones romanas', de Carlos Fernández Casado, publicado en Mayo de 1977”, Revista de Obras Públicas, 124, 725-727 (http://ropdigital.ciccp. es/pdf/publico/1977/1977_septiembre_3149_05.pdf).

SÁnchez Abal, J. L. (1977): "Obra hidráulica romana en la Provincia de Toledo (Pantano de Alcantarilla)". En Segovia. Sympósium de Arqueología Romana. Barcelona: Instituto de Arqueología y Prehistoria, Univ. de Barcelona, pp. 359-366.

SÁnchez-Chiquito, S. (coord.) (2005): Arqueología romana en Toletum: 1985-2004. Puesta en valor de las termas romanas de la plaza de Amador de los Ríos. Toledo: Consorcio de la ciudad de Toledo.

Sánchez-Palencia, F. J. (coord.) (1996): Toledo. Arqueología en la ciudad. Toledo: Junta de Comunidades de Castilla-La Mancha.

Silva, A. (2002): "Un nuevo tramo de la conducción hidráulica Proserpina-Los Milagros”, Mérida. Excavaciones arqueológicas 2000: Memoria 6, pp. 289-296.

Ventura, A. (1993): El abastecimiento de agua a la Córdoba romana. I: El acueducto de Valdepuentes. Córdoba: Univ. de Córdoba. 